\title{
Potentialité des données satellitaires Sentinel-2 pour la cartographie de l'impact des feux de végétation en Afrique tropicale : application au Togo
}

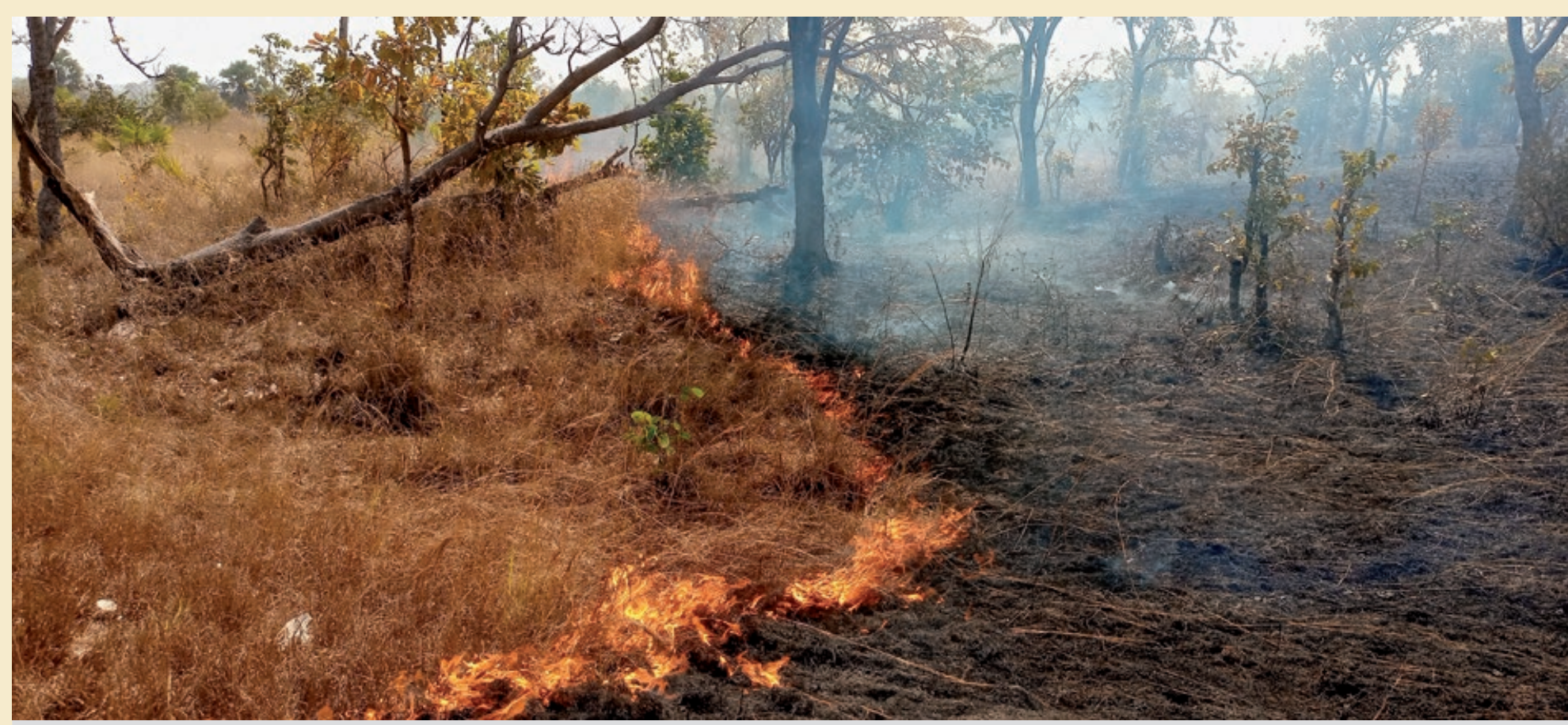

Photo 1.

Feux de végétation en zone savanicole dans la région de la Kara, au Togo.

Vegetation fires in the savannah zone in the Kara Region, Togo.

Photo Y. Konko.

\section{Yawo KonKO 1,2}

\section{Bareremna AfELU ${ }^{3}$}

\section{Kouami KoкоU ${ }^{4}$}

${ }^{1}$ Observatoire national de l'environnement Agence nationale de gestion de l'environnement (ANGE) Lomé

Togo

\begin{abstract}
${ }^{2}$ West African Science Service Centre on Climate Change

and Adapted Land Use (WASCAL)

Federal University of Technology

Minna

Nigeria

${ }^{3}$ Ministère de l'Environnement,

\author{
${ }^{4}$ Université de Lomé \\ Faculté des sciences \\ Laboratoire de recherche forestière \\ Lomé \\ Togo
} du Développement durable et de la Protection de la Nature (MEDDPN) Lomé Togo
Auteur correspondant / Corresponding author: Yawo KONKO - konkoyawo2@gmail.com

Doi : 10.19182/bft2021.347.a36349 - Droit d'auteur (c 2021, Bois et Forêts des Tropiques - (c) Cirad - Date de soumission : 16 juin 2020 ; date d'acceptation : 16 décembre 2020 ; date de publication : 25 février 2021.

\section{Ccirad (a)}

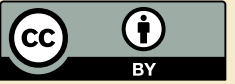

Citer l'article / To cite the article

Konko Y., Afelu B., Kokou K., 2021. Potentialité des données satellitaires Sentinel-2 pour la cartographie de l'impact des feux de végétation en Afrique tropicale : application au Togo. Bois et Forêts des Tropiques, 347: 59-75. Doi : https://doi.org/10.19182/bft2021.347.a36349 


\section{RÉSUMÉ}

\section{Potentialité des données satellitaires Sentinel-2 pour la cartographie de l'impact des feux de végétation en Afrique tropicale : application au Togo}

Le réchauffement climatique est un phénomène d'envergure mondiale qui se répercute sur le système climatique. Une des conséquences du réchauffement climatique est l'extension de la période de sécheresse, favorisant ainsi l'augmentation des fréquences du phénomène des feux de végétation. Les feux incontrôlés perturbent l'écologie et la fonctionnalité des écosystèmes, entraînant parfois leur érosion. La présente étude est une contribution pour la gestion des feux de végétation au Togo et porte sur le suivi spatial pour la saison des feux 2018-2019. Elle explore la potentialité des nouvelles données satellitaires Sentinel-2 (S-2) en accès libre dans la gamme de la télédétection optique pour la détection des surfaces brûlées, la cartographie des feux utilitaires et des feux incontrôlés. Elle teste également la performance de la méthode de cartographie des feux de végétation à partir de l'indice NBR (Normalized Burn Ratio) initialement conçue pour les images Landsat et évalue la biomasse végétale brûlée. Les résultats révèlent que les images $S-2$ présentent du potentiel dans la restitution des surfaces brûlées. La performance de la méthode de l'indice NBR sur les images S-2 est satisfaisante. La cartographie des feux de végétation montre que les feux utilitaires représentent $21,75 \%$ contre $78,25 \%$ pour les feux incontrôlés. L'ensemble des feux de végétation enregistrés a occasionné l'incendie de $5878 \mathrm{~km}^{2}$ du couvert végétal, soit $10,39 \%$ du territoire national. Le couvert végétal brûlé est composé majoritairement de savanes $(33,12 \%)$, de cultures et jachères $(24,48 \%)$, de plantations $(14,59 \%)$, de forêts claires (14,43\%) et de forêts riveraines (13,02\%). Les résultats obtenus constituent des éléments tangibles pour le suivi, la sensibilisation, l'élaboration des plans d'aménagement, de prévention et de gestion des feux.

Mots-clés : feux de végétation, feux utilitaires, feux incontrôlés, indice NBR, image Sentinel-2, occupation du sol, télédétection, Togo.

\section{ABSTRACT}

\section{Potential of Sentinel-2 satellite data to map the impacts of bush fires in tropical Africa: application in Togo}

Climate warming is a global phenomenon which is affecting the whole climate system. One of its consequences is the longer duration of drought periods, which is increasing the frequency of bush fires. Uncontrolled wildfires disrupt the ecology and functions of ecosystems, sometimes eroding them. This study, focusing on satellite monitoring during the bush fire season in 2018-2019, offers a contribution to support bush fire management in Togo. We explore the potential of new satellite data from Sentinel-2 (S-2), available as open-access remote sensing data for optical detection of burned areas and mapping of both utilitarian and uncontrolled fires. The data can also be used to test the performance of bush fire mapping methods by means of the NBR index (Normalized Burn Ratio), initially designed for Landsat images to assess burned plant biomass. The results show the potential usefulness of S-2 images in mapping burned areas and a satisfactory performance of the NBR method with S-2 images. Mapping of bushfires shows that utilitarian fires account for $21.75 \%$ of all fires as opposed to $78.25 \%$ for uncontrolled fires. Together, the bush fires recorded destroyed plant cover over $5,878 \mathrm{~km}^{2}$, or $10.39 \%$ of Togo's surface area. The plant cover burned mainly comprises savannah land (33.12\%), crops and fallows (24.48\%), plantations (14.59\%), open woodland (14.43\%) and riparian forest $(13.02 \%)$. The results obtained provide tangible evidence to support monitoring, awareness-raising, planning and development of bush fire prevention and management plans.

Keywords: bush fires, utilitarian fires, uncontrolled fires, NBR index, Sentinel-2 image, land use, remote sensing, Togo.

\section{RESUMEN}

\section{Potencial de los datos del satélite Sentinel-2 para cartografiar el impacto de los fuegos de vegetación en el África tropical: aplicación en Togo}

El calentamiento global es un fenómeno mundial que afecta al sistema climático. Una de las consecuencias del calentamiento global es la extensión del periodo de sequía, lo que aumenta la frecuencia de los fuegos de vegetación. Los incendios no controlados causan perturbaciones en la ecología y en las funciones de los ecosistemas, en ocasiones erosionándolos. El presente estudio es una contribución a la gestión de los fuegos de vegetación en Togo y se centra en el seguimiento espacial de la temporada de incendios 2018-2019. Explora el potencial de los nuevos datos del satélite Sentinel-2 (S-2), de acceso libre en el ámbito de la teledetección óptica, para localizar superficies quemadas y cartografiar los fuegos útiles y los incendios no controlados. También comprueba el rendimiento del método de cartografía de fuegos de vegetación mediante el índice NBR (Normalized Burn Ratio, índice de quema normalizado) desarrollado originariamente para las imágenes Landsat, y evalúa la biomasa vegetal quemada. Los resultados revelan que las imágenes del S-2 pueden ser útiles para restaurar las superficies quemadas. El rendimiento del método del índice NBR en las imágenes del S-2 es satisfactorio. La cartografía de los fuegos de vegetación muestra que los fuegos útiles representan el $21,75 \%$, frente al $78,25 \%$ para los incendios no controlados. El conjunto de los fuegos de vegetación registrados quemó 5878 km² de cubierta vegetal, es decir, el 10,39\% del territorio nacional. La cubierta vegetal quemada era principalmente sabana (33,12\%), cultivos y barbechos $(24,48 \%)$, plantaciones (14,59\%), bosques abiertos (14,43\%) y bosques de ribera $(13,02 \%)$. Los resultados obtenidos constituyen elementos tangibles para el seguimiento, la sensibilización y la elaboración de planes de ordenación, prevención y gestión de incendios.

Palabras clave: fuegos de vegetación, fuegos útiles, incendios no controlados, índice NBR, imagen del Sentinel-2, uso del suelo, teledetección, Togo. 
Le changement climatique est un phénomène d'envergure mondiale qui affecte de nombreux écosystèmes naturels (IPCC, 2018). Le réchauffement climatique issu des émissions de gaz à effet de serre (GES) augmente sans cesse le nombre d'événements météorologiques extrêmes tels que les pluies diluviennes et la sécheresse. Cette dernière est un contributeur clé aux phénomènes des feux de végétation qui engendrent des impacts coûteux sur l'agriculture, les moyens de subsistance et la santé humaine.

À l'échelle globale, l'évaluation récente basée sur les satellites d'observation de la Terre estime qu'environ 4 millions de $\mathrm{km}^{2}$ sont brûlés dans le monde chaque année (Chuvieco et al., 2018). Les feux de végétation sont ainsi considérés comme l'un des facteurs affectant la chimie atmosphérique à travers les émissions de gaz à effet de serre et d'aérosols (Knorr et al., 2016). Au niveau des écosystèmes forestiers, les feuxaffectent le bilan carbone (Yue etal., 2015), les structures horizontales et verticales des peuplements (Bowman et al., 2009) et demeurent un moteur important de transformation spatiale (Lewis et al., 2015). Dans les zones densément peuplées, les feux de végétation ont des impacts négatifs sur la qualité de l'air avec des effets connexes sur la santé des enfants et des personnes ayant des maladies cardiaques et des problèmes respiratoires (Reid et al., 2016). Au-delà de la végétation, ces feux génèrent des victimes humaines et animales lors de la propagation des incendies (Doerr et al., 2006 ; Chuvieco et al., 2010).

Le continent africain n'est pas épargné par le phénomène des feux de végétation. En Afrique tropicale, bien que les feux de végétation soient une pratique ancestrale dans l'agriculture et la gestion des terres (Sow et al., 2013 ; Kamau et Medley, 2014), le réchauffement climatique a grandement contribué à l'augmentation de ce phénomène à travers l'extension de la période de sécheresse et de celle de canicule.

Dans de nombreux pays d'Afrique de l'Ouest comme le Togo, il existe deux types de feux de végétation : les feux utilitaires et les feux incontrôlés. Selon la loi togolaise $n^{\circ} 2008$ 09 du code forestier, les feux utilitaires désignent les surfaces brûlées inférieures à 0,5 ha alors que les feux incontrôlés représentent les surfaces brûlées supérieures ou égales à 0,5 ha. Au Togo, les feux de végétation sont très courants. Mais, au-delà de certaines normes, les feux utilitaires pour l'agriculture, le pastoralisme, le renouvellement de la paille et le nettoiement des champs échappent au contrôle humain et deviennent des feux incontrôlés qui ont des impacts nocifs pour les écosystèmes forestiers, l'environnement et les populations. Selon certains auteurs, les feux incontrôlés dégradent les écosystèmes, modifient le paysage et perturbent la tranquillité de la biocénose avec des conséquences importantes pour la survie et l'écologie des habitats (Konko, 2016 ; Chuvieco et al., 2019). Pour documenter ces phénomènes, les systèmes de contrôle et de suivi des feux de végétation constituent des moyens idéaux pour une meilleure gestion de l'environnement. Selon plusieurs auteurs, la télédétection est un outil opérationnel pour le suivi des feux de végétation (Afelu, 2016 ; Mpakairi et al., 2020).
La télédétection se présente comme un des meilleurs outils pour la surveillance terrestre (Konko et al., 2020). En effet, elle a la capacité d'observer une même unité spatiale sur plusieurs intervalles de temps et fournit des informations spatio-temporelles relatives (González et al., 2013). La télédétection offre ainsi un potentiel considérable pour surveiller les feux de végétation. Au cours des dernières décennies, la télédétection a été largement utilisée pour surveiller les zones brûlées à l'échelle mondiale. Plusieurs capteurs embarqués sur des satellites enregistrent les émissions thermiques et la réflectance spatiale afin de produire des données pour le suivi des feux. Parmi les capteurs les plus utilisés pour la cartographie des feux, on peut citer les capteurs Thematic Mapper (TM ; Veraverbeke et al., 2010b ; Bastarrika et al., 2011), Enhanced Thematic Mapper plus (ETM + ; Smith et al., 2007 ; Bastarrika et al., 2011), Operational Land Imager (OLI ; Schroeder et al., 2016), Advanced Spaceborne Thermal Emission and Reflection Radiometer (ASTER ; Stroppiana et al., 2009 ; Boschetti et al., 2010), Moderate Resolution Imaging Spectroradiometer (MODIS ; Smith et al., 2007), VEGETATION (VGT ; Lasaponara, 2006 ; Bartalev et al., 2007), Advanced Very High Resolution Radiometer (AVHRR ; Chuvieco et al., 2008 ), MutiSpectral Instrument (MSI ; Mpakairi et al., 2020).

Partant des capteurs TM, ETM+ et OLI des satellites Landsat (Landsat-1 à Landsat-8) de la NASA (National Aeronautics and Space Administration) aux capteurs MSI des satellites Sentinel-2 (S-2) de l'ESA (European Space Agency), les gestionnaires disposent de nos jours d'une multitude de données d'imagerie satellitaire en accès libre et gratuit.

S'agissant des données Landsat, le temps de revisite du satellite de 16 jours (Images OLI et TIRS) ne permet pas d'optimiser les chances de disposer de plusieurs images dans un temps rapproché pour un suivi et pour éviter le problème induit par la présence de nuages, récurrent en contexte tropical. De plus, la résolution de $30 \mathrm{~m}$ de ces images constitue une limite pour la cartographie des petites surfaces brûlées. Le développement du programme Copernicus de l'ESA depuis 2015 offre de nouvelles images optiques S-2 avec des améliorations de la résolution spatiale (10 à $60 \mathrm{~m})$ et sur le temps de revisite des satellites ( 5 jours). Grâce à leurs résolutions spatiales, les images S-2 peuvent faire la distinction entre les feux utilitaires et les feux incontrôlés. Les satellites fournissent plusieurs images $\mathrm{S}-2$ dans un temps court, favorisant ainsi le suivi de la végétation et une meilleure gestion des problèmes induits par la présence de nuages.

Cependant, bien que les images S-2 offrent de meilleures caractéristiques, très peu de travaux de recherche ont été réalisés pour évaluer leur potentiel dans le suivi des feux de végétation des écosystèmes tropicaux terrestres (Verhegghen et al., 2016). La présente étude a été entreprise dans le souci de contribuer à une meilleure connaissance du potentiel des images S-2 pour le suivi des feux de végétation en Afrique tropicale. Elle a eu pour objectif général d'explorer, sur le Togo, les potentialités des images S-2 pour la surveillance et la cartographie des feux de végétation. 


\section{Matériels et méthode}

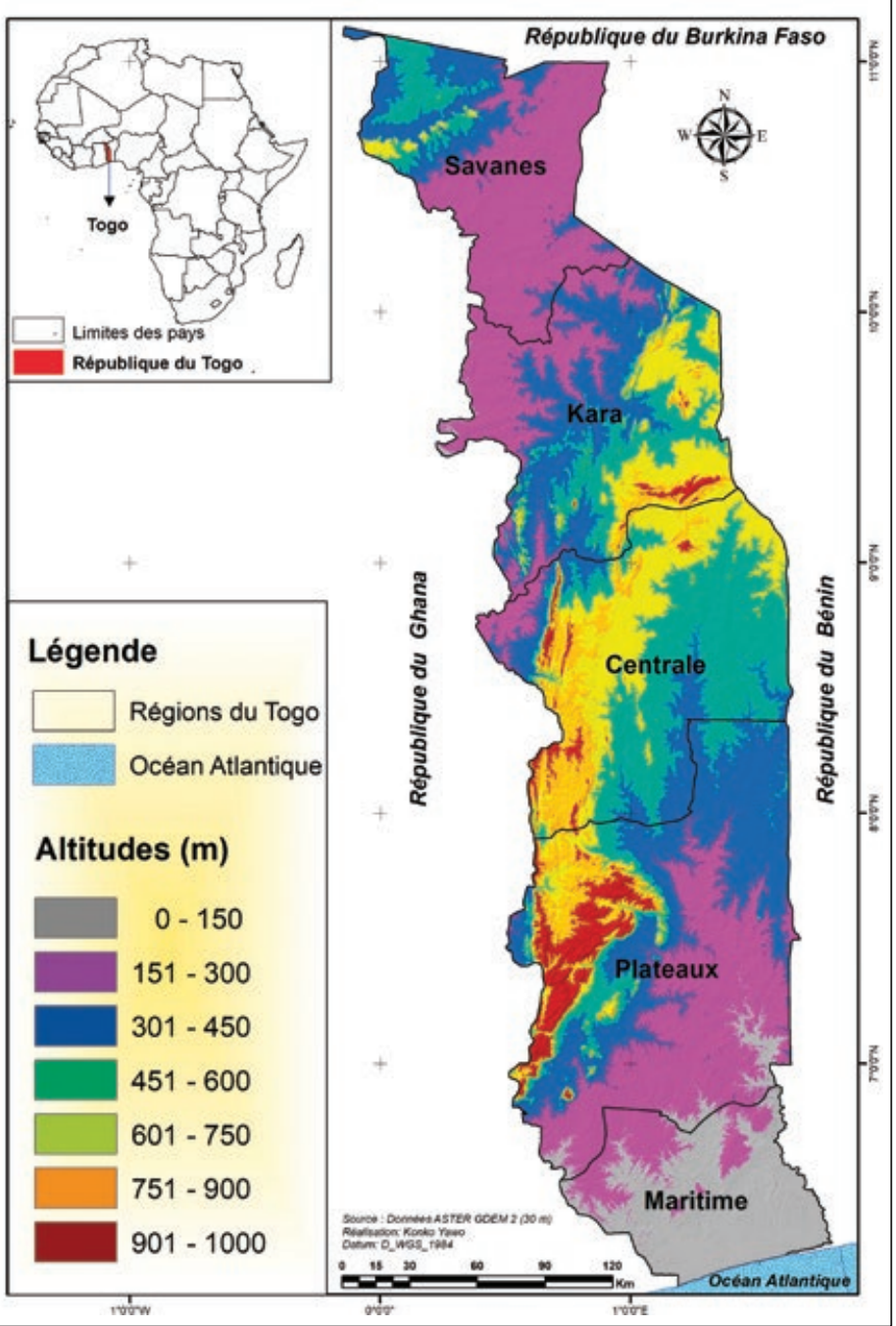

Figure 1.

Situation géographique du Togo montrant les régions et le modèle numérique d'altitude. Geographical location of Togo showing the regions and the digital elevation model.
Quatre objectifs spécifiques ont été déclinés pour cette étude. Le premier était d'évaluer la potentialité des images S-2 dans la restitution des surfaces brûlées. Quant au deuxième, sur la base des images S-2, il s'agissait de tester la performance de la méthode de cartographie des surfaces brûlées suivant les classes d'occupation du sol à partir de l'indice NBR (Normalized Burn Ratio) retenu à cause de sa simplicité de mise en œuvre et de sa robustesse (Veraverbeke et al., 2012). Le troisième objectif était de procéder à la cartographie des feux utilitaires et des feux incontrôlés. Enfin, le quatrième consistait à cartographier les classes du couvert végétal brûlé assorti des analyses statistiques. Ce dernier objectif permettrait d'identifier les classes du couvert végétal brûlé.

\section{Zone d’étude}

La zone d'étude de cette recherche se situe en République du Togo sur la côte du golfe de Guinée en Afrique de l'Ouest. Le Togo a une superficie de 56600 km². Il est limité au sud par l'océan atlantique, au nord par le Burkina Faso, à l'est par le Bénin et à l'ouest par le Ghana (Nimon et al., 2020). Le relief du Togo est formé de vastes plaines et de nombreuses collines. Le plus haut sommet est la montagne d'Agou atteignant une altitude de 986 m (figure 1).

Le Togo appartient à la zone intertropicale marquée de deux grands régimes climatiques. Le régime tropical soudanien au nord avec une saison pluvieuse et une saison sèche. Le régime tropical guinéen au sud, caractérisé par deux saisons sèches et deux saisons pluvieuses de durées inégales (Nimon et al., 2020).

Le territoire national est divisé en cinq régions administratives et économiques : région Maritime (6 $100 \mathrm{~km}^{2}$ ), région des Plateaux (16 $\left.975 \mathrm{~km}^{2}\right)$, région Centrale (13 $\left.317 \mathrm{~km}^{2}\right)$, région de la Kara $\left(11738 \mathrm{~km}^{2}\right)$, région des Savanes $\left(8470 \mathrm{~km}^{2}\right)$. Selon, les résultats définitifs du quatrième Recensement général de la population et de l'habitat (RGPH) réalisé du 6 au 21 novembre 2011, la population du Togo était estimée à 6191155 habitants.

Outre les régions administratives et économiques, le Togo est subdivisé en cinq zones écologiques. Ces zones sont essentiellement couvertes de savanes (Guelly, 1994). Quelques massifs et îlots forestiers apparaissent par endroits à la faveur de l'humidité, que ce soit en terre ferme, en altitude ou le long des cours d'eau. La biodiversité est très diversifiée de par la variété des écosystèmes aquatiques (cours d'eau, lacs, marécages, eaux maritimes) et terrestres, à savoir les savanes guinéennes et soudaniennes et les forêts (Ern, 1979). On y rencontre les espèces animales inférieures et supérieures, des protozoaires aux vertébrés. Selon le cinquième rapport national sur la diversité biologique du Togo (2009-2014), un total de 4019 espèces animales a été recensé contre 3700 en 2009, soit une augmentation de $8 \%$.

\section{Données utilisées}

Les données satellitaires utilisées dans cette étude sont des images optiques S-2 en accès libre. Elles sont disponibles sur la plateforme de l'ESA. Les images S-2 ont été acquises par les satellites Sentinel-2A et Sentinel-2B équipés d'un capteur MSI (Multi Spectral Instrument). Le capteur MSI mesure le rayonnement réfléchi de la Terre dans 13 bandes spectrales et dispose d'une résolution spatiale allant de 10 à $60 \mathrm{~m}$. Pour cette étude, les données S-2 acquises avant et après le passage du feu ont été utilisées. La période du $1^{\text {er }}$ novembre 2018 au 30 avril 2019 correspond à la saison des feux de végétation. Le choix des images a été fait sur la base de la méthode du plus proche voisin afin de retenir la meilleure image avec une couverture nuageuse minimale. Le temps de revisite de 5 jours des satellites S-2 a 
Tableau I.

Caractéristiques des images S-2 utilisées.

Characteristics of the S-2 images used.

\begin{tabular}{|c|c|c|c|c|c|}
\hline \multirow[t]{2}{*}{ Satellite } & \multirow[t]{2}{*}{ Capteur } & \multicolumn{2}{|c|}{$\begin{array}{l}\text { Dates d'acquisition des images } \\
\text { (année_mois_jour) }\end{array}$} & \multirow[t]{2}{*}{$\begin{array}{l}\text { Résolution } \\
\text { spatiale }(\mathrm{m})\end{array}$} & \multirow[t]{2}{*}{$\begin{array}{l}\text { Numéro de } \\
\text { tuile au sol }\end{array}$} \\
\hline & & $\begin{array}{l}\text { Image avant le } \\
\text { passage du feu }\end{array}$ & $\begin{array}{l}\text { Image après le } \\
\text { passage du feu }\end{array}$ & & \\
\hline \multirow[t]{15}{*}{ Sentinel-2 } & \multirow[t]{15}{*}{ MSI } & 2018_11_30 & 2019_04_04 & $10(10-60)$ & T_31PBM \\
\hline & & 2018_11_08 & 2019_03_28 & $10(10-60)$ & T_31PBN \\
\hline & & 2018_11_23 & 2019_03_28 & $10(10-60)$ & T_30PZS \\
\hline & & 2018_11_23 & 2019_03_28 & $10(10-60)$ & T_30PZT \\
\hline & & 2018_11_23 & 2019_03_28 & $10(10-60)$ & T_30PZR \\
\hline & & 2018_11_30 & 2019_04_09 & $10(10-60)$ & T_31PCL \\
\hline & & 2018_11_30 & 2019_04_04 & $10(10-60)$ & T_31PBL \\
\hline & & 2018_11_30 & 2019_04_09 & $10(10-60)$ & T_31PBK \\
\hline & & 2018_11_30 & 2019_03_30 & $10(10-60)$ & T_31PCK \\
\hline & & 2018_11_30 & 2019_03_05 & $10(10-60)$ & T_31NBJ \\
\hline & & 2018_11_30 & 2019_03_05 & $10(10-60)$ & T_31NCJ \\
\hline & & 2018_12_25 & 2019_04_09 & $10(10-60)$ & T_31NBH \\
\hline & & 2018_12_25 & 2019_04_09 & $10(10-60)$ & T_31NCH \\
\hline & & 2018_12_25 & 2019_04_09 & $10(10-60)$ & T_31NBG \\
\hline & & 2018_12_25 & 2019_04_09 & $10(10-60)$ & T_31NCG \\
\hline
\end{tabular}

permis d'avoir une série d'images dans un temps rapproché suffisante pour orienter le choix et contourner le problème des nuages. Les caractéristiques des images utilisées sont présentées dans le tableau I.

Par ailleurs, les données de l'occupation du sol de 2013-2014 issues du traitement des images satellitaires Rapideye à $5 \mathrm{~m}$ de résolution spatiale fournies par le ministère de l'Environnement ainsi que les données topographiques nationales fournies par la Direction générale de la cartographie à l'échelle $1 / 50000^{\circ}$ ont également été retenues comme données de référence.

\section{Traitements des images satellitaires}

\section{Prétraitement des images satellitaires}

Dans le cadre de cette étude, seules les bandes B2 $(\lambda=0,490 \mu \mathrm{m}), \mathrm{B} 3(\lambda=0,560 \mu \mathrm{m}), \mathrm{B} 4(\lambda=0,665 \mu \mathrm{m}), \mathrm{B} 8$ $(\lambda=0,842 \mu \mathrm{m})$ et B12 $(\lambda=2,190 \mu \mathrm{m})$ des images S-2 ont été nécessaires pour le traitement. Le logiciel SNAP (Sentinel Application Platform) destiné au traitement des images S-2 a été adopté. Le temps de revisite de 5 jours a constitué un avantage clé pour bénéficier d'une série d'images faiblement ennuagées dans un laps de temps court. La saison des feux de végétation coïncidant avec la saison sèche a également augmenté les chances d'obtenir des images sans nuages. Pour ces raisons, le prétraitement a porté essentiellement sur les opérations de correction radiométrique, de rééchantillonnage de bande, de mosaïquage des bandes et de composition colorée.

La correction radiométrique a servi pour la réduction des effets atmosphériques et le rehaussement de contraste des scènes par la méthode de MERIS avec l'algorithme de correction de Rayleigh (Schroeder et al., 2007). L'opération de rééchantillonnage de bande a harmonisé la résolution spatiale de la bande B12 sur la résolution spatiale de $10 \mathrm{~m}$ des autres bandes B2, B3, B4 et B8 en utilisant l'algorithme "Nearest neighbour ». Cet algorithme a l'avantage de préserver les valeurs d'origine des scènes. Les bandes de $10 \mathrm{~m}$ ont été retenues pour le rééchantillonnage afin de permettre la cartographie des superficies brûlées inférieures à 0,5 ha (feux utilitaires). Le mosaïquage des bandes a lié les mêmes numéros de bande d'images en une bande unique couvrant tout le Togo. Les bandes d'images avant le passage du feu et après le passage du feu ont été mosaïquées séparément par l'outil «Mosaicing ». La composition colorée de bandes en vraies couleurs (proche de la réalité visuelle humaine) a été produite pour permettre une visualisation en couleurs naturelles des images, afin de mieux discriminer la végétation non brûlée et les surfaces brûlées. Cette composition a été effectuée en associant la bande B4 au canal rouge, la bande B3 au canal vert et la bande B2 au canal bleu.

\section{Création de masque d'eau}

Les zones aquatiques sont le plus souvent confondues avec les surfaces brûlées sur les indices de végétation utilisés pour la cartographie des feux de végétation (Mallinis et al., 2018). Afin de lever cette confusion, il a été nécessaire de générer un masque pour séparer les zones aquatiques des surfaces brûlées. Plusieurs masques pour la discrimination des zones aquatiques (fleuves, rivières, lacs, lagunes, plans d'eau, etc.) à partir de l'imagerie satellitaire ont été produits et appliqués tels que le NDWI (Normalized Difference Water Index) (McFeeters, 1996) et le MNDWI (Modified Normalized Difference Water Index) (Wang et al., 2017). 
Pour cette étude, l'indice NDWI a été choisi en raison de son efficacité, sa popularité et sa commodité. Le NDWI est calculé ainsi :

$N D W I=\frac{\text { Vert }-P I R}{\text { Vert }+P l R}($ équation 1$)$

où le PIR (proche Infrarouge) est la bande B8 et le vert la bande B3.

Le NDWI maximise la réflectance de l'eau et minimise la réflectance de la végétation et du sol, permettant ainsi une meilleure discrimination des zones aquatiques. En règle générale, avec le NDWI l'eau a des valeurs positives tandis que le sol et la végétation ont une valeur nulle ou négative.

\section{Production des indices NBR avant et après le passage du feu}

Plusieurs indices de végétation pour la cartographie des feux de végétation à partir de l'imagerie satellitaire ont été calculés et appliqués : l'indice NDVI (Normalized Difference Vegetation Index) (Tucker, 1979), l'indice BAl (Burned Area Index) (Chuvieco et al., 2002), l'indice NBR (Normalized Burn Ratio) (Key and Benson, 2006) et l'indice CSI (Char Soil Index) (Smith et al., 2007). Dans le cadre de cette étude, l'indice NBR a été retenu à cause de sa simplicité, sa robustesse et sa capacité de mesurer la sévérité des brûlages (Veraverbeke et al., 2012). L'indice NBR avant le passage du feu et l'indice NBR après le passage du feu sont calculés successivement :

$N B R=\frac{P I R-S W I R}{P I R+S W I R}($ équation 2)

où le PIR fait référence à la bande B8 et le SWIR (infrarouge à ondes courtes) à la bande B12.

Étant donné que l'indice NBR est conçu initialement pour être utilisé sur les images Landsat, sa performance sur les images S-2 a été testée en premier lieu.

\section{Seuillage du delta NBR}

L'indice NBR avant le passage du feu et l'indice NBR après le passage du feu obtenus à partir des images $\mathrm{S}-2$ ont été utilisés pour calculer le delta NBR $(\triangle N B R)$, lequel permet la mesure de la sévérité des brûlages (Miller et al., 2009 ; Veraverbeke et al., 2010a ; García-Llamas et al., 2019). La formule utilisée pour calculer le $\triangle \mathrm{NBR}$ est la suivante :

$$
\triangle N B R=N B R_{\text {Avant feux }}-N B R_{\text {Après feux }} \text { (équation 3) }
$$

Une valeur élevée de $\triangle N B R$ indique des dommages plus graves, tandis que les zones avec des valeurs faibles voire négatives peuvent signifier une surface brûlée ou une repousse après un feu. Pour cette étude, la classification fournie par le portail USGS pour l'interprétation de la sévérité des brûlages a été adoptée avec quelques modifications. Selon cette classification, la sévérité des brûlages est considérée comme faible si $\triangle N B R$ est compris entre 0,1 et 0,269 , faiblement modérée lorsque $\triangle N B R$ est compris entre 0,27 et 0,439 , hautement modérée si $\triangle N B R$ est compris entre 0,44 et 0,659 et hautement sévère si $\Delta N B R$ est compris entre 0,66 et 1,3 . Pour cette étude, les valeurs de $\triangle N B R$ supérieures au seuil de 0,27 ont été extraites et classées comme " surfaces brûlées » avec la méthode de binarisation après vérification et validation des données de terrain. Ceci se justifie par le fait que la législation togolaise ne dispose pas d'une hiérarchisation pour la sévérité des brûlages.

\section{Calcul des superficies brûlées}

L'indice $\triangle N B R$ binaire a permis de séparer la classe des surfaces brûlées de la classe des surfaces non brûlées. Le masque d'eau (NDWI) a été utilisé par la même occasion pour exclure la confusion entre les zones aquatiques et les zones brûlées. Par la suite, l'indice $\triangle N B R$ a été vectorisé afin de rendre possible la création des fichiers de forme (shapefile) (Konko, 2016 ; Atsri et al., 2018) et le calcul des superficies brûlées. Enfin, deux cartes de surfaces brûlées ont été générées. La première est appelée carte des feux utilitaires dont les superficies brûlées sont inférieures à 0,5 ha et la seconde représente la carte des feux incontrôlés avec des superficies brûlées supérieures ou égales à 0,5 ha. Cette catégorisation s'explique par le fait que la loi de la République togolaise décrit dans son code forestier $n^{\circ}$ 2008-09, articles 26 et 27 , deux types de feux de végétation : les feux utilitaires (feux de cultures agricoles et pastorales, de renouvellement de la paille et de nettoiement des champs) et les feux incontrôlés ou incendies de forêts dont les superficies brûlées sont supérieures ou égales à 0,5 ha.

\section{Cartographie du couvert végétal brûlé}

Les shapefiles des surfaces brûlées par les feux utilitaires et les incendies de forêts ont été utilisés pour extraire les classes du couvert végétal brûlé à partir du shapefile de l'occupation du sol de référence fourni par le ministère de l'Environnement du Togo. La technique du " cliping » a été adoptée pour cette opération. Par la suite, les superficies ont été calculées à l'aide de la table attributaire.

\section{Méthode de validation des résultats}

Sur le plan scientifique, les résultats des traitements des images satellitaires ne deviennent véritablement fiables qu'après une validation menée sur le terrain (ou avec des données de référence) et lorsqu'elles sont accompagnées de statistiques et d'indicateurs pertinents de qualité des résultats. Dans le cadre de cette étude, une première validation a été réalisée pour évaluer la performance du seuil du $\triangle$ NBR retenu suivant les classes d'occupation du sol et une seconde validation pour l'ensemble des résultats obtenus à l'issue des traitements. Au total, 683 points ont été retenus pour les contrôles au sol. La distribution des points dans différentes classes d'occupation du sol brûlé a été faite de manière aléatoire avec une contrainte de distance de $100 \mathrm{~m}$ entre les points voisins pour éviter toute corrélation (Congalton, 1991). À l'issue des vérifications de terrain, une matrice de confusion a été produite (tableau II).

Cette matrice a servi de soubassement pour le calcul des différents indicateurs de précision jugés pertinents, notamment la précision globale, la précision du producteur, la précision de l'utilisateur, la précision F-score, et les erreurs de commission et d'omission. Ces différents indicateurs ont été retenus car ils sont considérés comme plus pertinents que l'indice Kappa pour les validations détaillées des produits cartographiques (Story et Congalton, 1986 ; Rwanga et Ndambuki, 2017 ; Stehman et Foody, 2019).

La précision globale (PG) indique le pourcentage des points bien classés par rapport au nombre total de points contrôlés. Elle représente le rapport entre le nombre total de 
Tableau II.

Matrice de confusion.

Confusion matrix.

\begin{tabular}{|c|c|c|c|c|c|c|c|c|c|}
\hline \multirow[b]{2}{*}{ Classes brûlées } & \multirow[b]{2}{*}{$\begin{array}{l}\text { Forêts } \\
\text { denses }\end{array}$} & \multirow[b]{2}{*}{$\begin{array}{l}\text { Forêts } \\
\text { riveraines }\end{array}$} & \multirow[b]{2}{*}{$\begin{array}{l}\text { Forêts } \\
\text { claires }\end{array}$} & \multirow[b]{2}{*}{ Plantations } & \multicolumn{2}{|c|}{ Vérités terrain } & \multirow[b]{2}{*}{$\begin{array}{c}\text { Zones } \\
\text { marécageuses }\end{array}$} & \multirow[b]{2}{*}{$\begin{array}{l}\text { Cultures } \\
\text { et jachères }\end{array}$} & \multirow[b]{2}{*}{ Total } \\
\hline & & & & & Savanes & $\begin{array}{c}\text { Parcs } \\
\text { agroforestiers }\end{array}$ & & & \\
\hline Forêts denses & 75 & 1 & 1 & 1 & 3 & 0 & 0 & 5 & 86 \\
\hline Forêts riveraines & 0 & 75 & 0 & 0 & 3 & 0 & 0 & 3 & 81 \\
\hline Forêts claires & 5 & 0 & 76 & 1 & 4 & 2 & 0 & 1 & 89 \\
\hline Parcs agroforestiers & 0 & 0 & 0 & 0 & 3 & 80 & 2 & 0 & 85 \\
\hline Zones marécageuses & 0 & 0 & 0 & 0 & 0 & 0 & 79 & 3 & 82 \\
\hline Cultures et jachères & 0 & 2 & 0 & 5 & 0 & 0 & 1 & 82 & 90 \\
\hline Total & 80 & 78 & 77 & 94 & 92 & 82 & 82 & 98 & 683 \\
\hline
\end{tabular}

points corrects ( $T_{\text {corrects }}$ ) et le nombre total de points contrôlés ( $T_{\text {contrôlés }}$ (Rwanga et Ndambuki, 2017). La formule utilisée pour calculer la PG est la suivante :

$P G=\frac{\text { (T corrects) }}{(T \text { contrôlés) }}$ (équation 4)

La précision du producteur (PP) est la probabilité pour un point appartenant à une classe de la vérité terrain d'être correctement classé. Contrairement à la précision globale, il s'agit d'un indicateur de précision par classe qui se calcule en faisant le rapport entre le nombre de points corrects de la colonne de la vérité terrain pour une classe $\left(\Delta_{\text {corrects }}\right)$ et le nombre total de points contrôlés pour la même colonne de classe $\left(\Delta_{\text {contrôlés }}\right)$ (Story et Congalton, 1986). L'équation 5 indique le calcul de la PP :

$P P=\frac{(\Delta \text { corrects) }}{(\Delta \text { contrôlés })}$ (équation 5$)$

Le complément de la précision de producteur (1 - PP) est l'erreur d'omission (Stehman et Foody, 2019). La précision de l'utilisateur (PU) est également un indicateur de précision par classe. Elle est la probabilité pour un point classé dans une classe sur la carte d'appartenir effectivement à cette classe sur le terrain. Elle représente le rapport entre le nombre de points corrects de la ligne de classe sur la carte $\left(U_{\text {corrects }}\right)$ et le nombre total de points contrôlés pour la même ligne de classe ( $\left.U_{\text {contrôlés }}\right)$ (Story et Congalton, 1986). La formule utilisée pour calculer la PU est la suivante :

$P U=\frac{(U \text { corrects) }}{(U \text { contrôlés })}$ (équation 6)

Le complément de la précision de l'utilisateur (1 - PU) est l'erreur de commission (Stehman et Foody, 2019). La précision F-Score (PFS) est l'indicateur de synthèse de la précision utilisateur et de la précision producteur pour évaluer la performance de la méthode de traitement. Elle est donnée par la moyenne géométrique de la précision producteur et de la précision utilisateur :

$P F S=\frac{(P P+P U)}{2}($ équation 7)

\section{Résultats}

\section{Les indicateurs de précision des résultats}

Les résultats de la cartographie des feux de végétation ont été validés à partir des données de terrain et de la matrice de confusion. Le tableau III présente les différents indicateurs de précision calculés à partir de la matrice de confusion.

La précision globale des résultats obtenus est de l'ordre de 92,53\%. Pour l'ensemble des classes d'occupation du sol, les précisions du producteur et de l'utilisateur sont supérieures à 80 \%. À l'exception de la classe cultures et jachères, les précisions $F$-Score ou les moyennes géométriques entre la précision producteur et la précision utilisateur sont supérieures à $90 \%$, indiquant un fort rapprochement des valeurs de la carte et des valeurs du terrain et par conséquent une cartographie de qualité. Les erreurs d'omission et de commission sont globalement faibles. Il découle de ces indicateurs de précision que la méthode d'analyse est bonne et que les résultats sont fiables et pourront être mis à disposition des décideurs ou utilisateurs pour exploitation.

\section{Potentialité des images S-2 dans la restitution des ressources en eau et la création de masque d'eau}

Le processus de traitement des images S-2 a permis de générer un masque d'eau à partir de l'indice NDWI. Ce masque a servi pour lever les confusions entre les zones aquatiques et les surfaces brûlées. La figure 2 est un extrait de scène couvrant un plan d'eau et ses alentours dans la région des Plateaux. La figure $2 \mathrm{a}$ représente l'extrait de l'image composite montrant le potentiel de restitution des plans d'eau sur les images $S$-2. Les figures $2 b$ et $2 c$ montrent respectivement l'extrait de l'indice NDWI et l'extrait du masque d'eau généré. Le masque d'eau présenté dans la 
Bois et Forêts des Tropiques - ISSN: L-0006-579X

Volume $347-1^{\text {st }}$ quarter - February 2021 - p. 59-75

FOCUS / BUSH FIRES DETECTION WITH SENTINEL- 2

\section{Tableau III.}

Les différents indicateurs de précision calculés.

The different precision indicators calculated.

\begin{tabular}{|c|c|c|c|c|c|}
\hline & & & Précisions & & \\
\hline Classes brûlées & $\begin{array}{c}\text { Précision } \\
\text { producteur (\%) }\end{array}$ & $\begin{array}{c}\text { Précision } \\
\text { utilisateur (\%) }\end{array}$ & $\begin{array}{c}\text { Erreur } \\
\text { d'omission (\%) }\end{array}$ & $\begin{array}{c}\text { Erreur de } \\
\text { commission (\%) }\end{array}$ & F-Score (\%) \\
\hline Forêts denses & 93,75 & 87,21 & 6,25 & 12,79 & 90,48 \\
\hline Forêts riveraines & 96,15 & 92,59 & 3,85 & 7,41 & 94,37 \\
\hline Forêts claires & 98,70 & 85,39 & 1,30 & 14,61 & 92,05 \\
\hline Plantations & 91,49 & 95,56 & 8,51 & 4,44 & 93,52 \\
\hline Savanes & 85,87 & 98,75 & 14,13 & 1,25 & 92,31 \\
\hline Parcs agroforestiers & 97,56 & 94,12 & 2,44 & 5,88 & 95,84 \\
\hline Zones marécageuses & 96,34 & 96,34 & 3,66 & 3,66 & 96,34 \\
\hline Cultures et jachères & 83,67 & 91,11 & 16,33 & 8,89 & 87,39 \\
\hline
\end{tabular}

figure $2 c$ est d'une bonne qualité et restitue suffisamment les éléments de l'indice NDWI, notamment le plan d'eau et ses contours ainsi que le fleuve Mono, ceci en conformité avec l'image composite (figure 2a). Cela témoigne non seulement de la qualité de l'indice NDWI à partir duquel le masque est généré d'une manière précise, mais également du potentiel des images S-2 dans la restitution des ressources en eau.

\section{Performance de la méthode de l'indice NBR sur les images S-2}

Outre le masque d'eau, l'indice NBR a été produit pour permettre la cartographie des feux de végétation. La figure 3 comprend plusieurs extraits de scènes couvrant la montagne de Bassar et ses alentours dans la région de la Kara. Parmi ces extraits figurent les différents NBR générés. Les figures $3 a, 3 b$ et $3 c$ montrent respectivement un extrait de l'indice NBR avant passage du feu, de l'indice NBR après le passage du feu et de l'indice $\triangle N B R$. L'interprétation visuelle de l'indice $\triangle N B R$ permet une identification des surfaces brûlées en couleur blanche.

Le produit de $\triangle$ NBR a été utilisé pour la cartographie des surfaces brûlées à travers l'extraction des valeurs de $\triangle$ NBR supérieures au seuil de 0,27 . Le résultat a été ensuite binarisé et vectorisé. Les figures $3 d$, $3 e$ et $3 f$ offrent respectivement un aperçu des valeurs de $\triangle N B R>0,27$ (surfaces brûlées) en rouge, de $\triangle N B R$ binaire montrant les valeurs de $\triangle N B R>0,27$ en rouge et de $\Delta N B R>0,27$ binaire vectorisé. La comparaison visuelle de ces trois figures illustre l'efficacité de la méthodologie utilisée pour l'extraction des surfaces brûlées. Cette efficacité est confirmée par l'indicateur de la précision globale qui est de l'ordre 92,53\%.

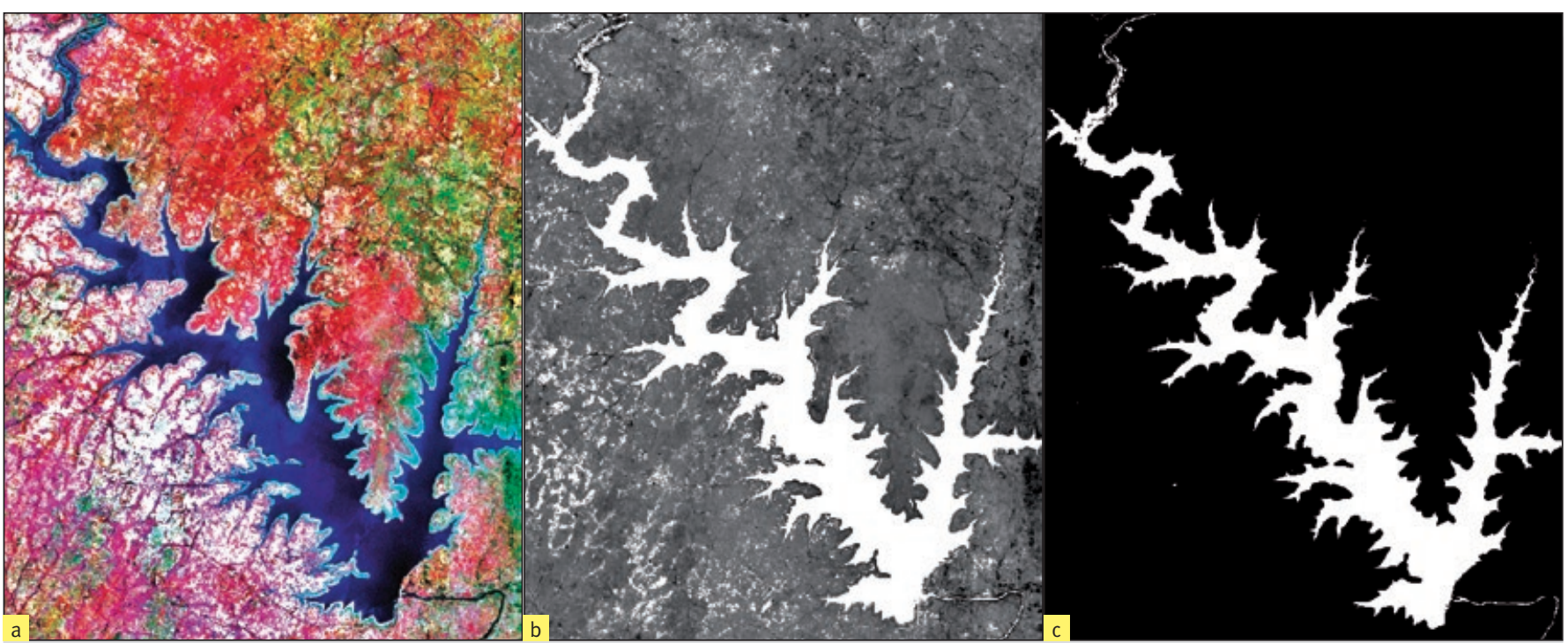

Figure 2.

Aperçu de la potentialité des images S-2 dans la restitution des ressources en eau et la création de masque. (a) Image composite du 30 novembre 2018 montrant un plan d'eau. (b) Indice NDWI. (c) Masque d'eau généré à partir de l'indice NDWI. Overview of the potential of $S-2$ images in the restitution of water resources and mask creation. (a) Composite image of 30 November 2018 showing a body of water. (b) NDWI index. (c) Water mask generated from the NDWI index. 


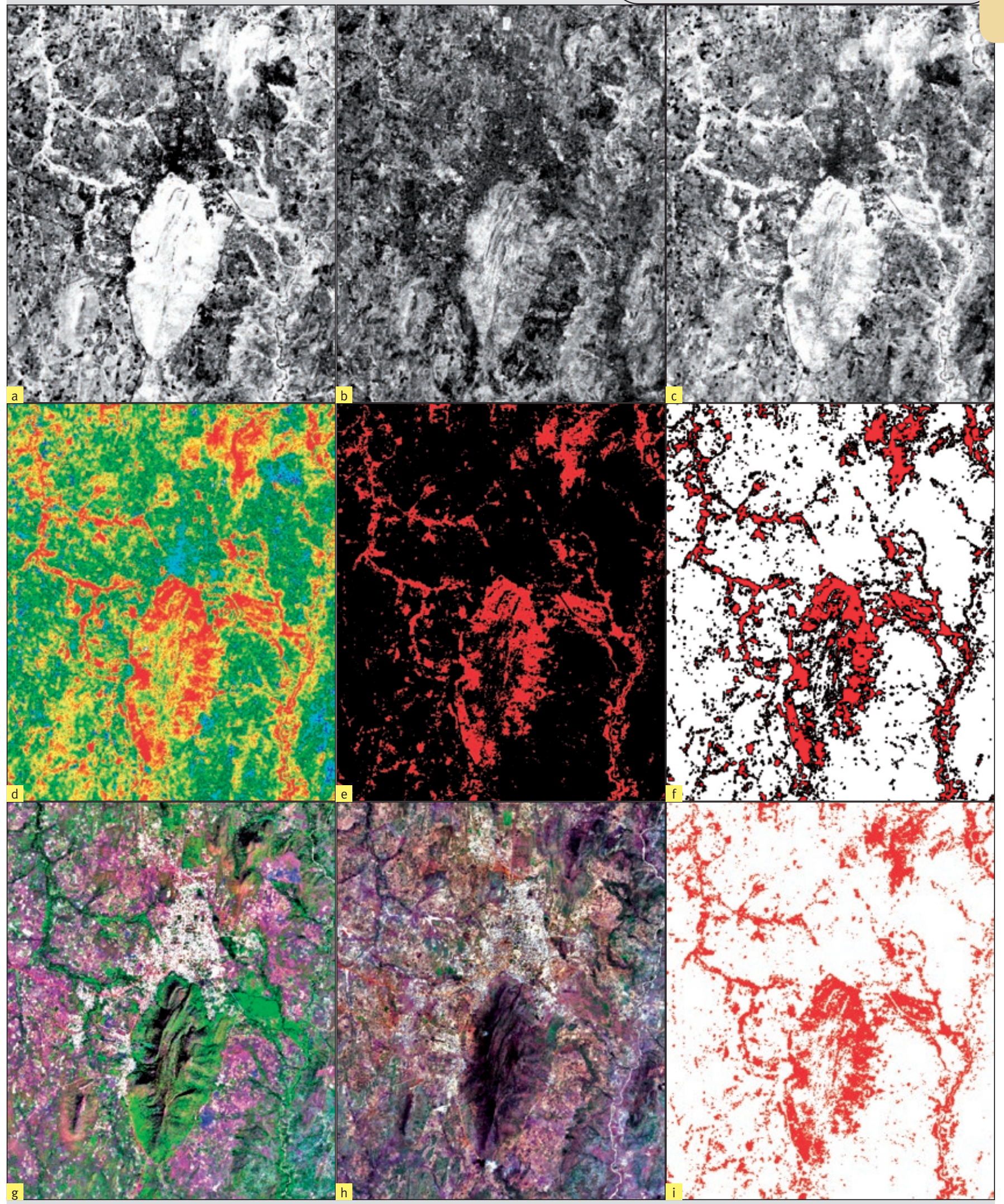

Figure 3.

Quelques extraits de scènes couvrant la montagne de Bassar et ses alentours dans la région de la Kara. (a) NBR avant le passage du feu. (b) NBR après le passage du feu. (c) $\triangle N B R$. (d) Image de $\triangle N B R$ montrant les valeurs de $\Delta N B R>0,27$ (surfaces brûlées) en rouge. (e) Image $\triangle N B R$ binaire montrant les valeurs de $\Delta N B R>0,27$ en rouge. (f) $\Delta N B R$ binaire vectorisé. (g) Image composite avant le passage du feu. (h) Image composite après le passage du feu. (i) Surfaces brûlées extraites en rouge. 


\section{Potentialité des images S-2 pour la cartographie des feux de végétation}

Désirant mettre en confiance les décideurs et les utilisateurs sur la qualité des données de la présente étude, des extraits de scènes ont été présentés côte à côte pour une appréciation visuelle de l'efficacité du résultat issu de la démarche méthodologique, en plus des indicateurs de précision. Les figures $3 \mathrm{~g}$, $3 \mathrm{~h}$ et $3 \mathrm{i}$ illustrent respectivement la situation avant le passage du feu, la situation après le passage de feu et les surfaces brûlées cartographiées. L'interprétation visuelle de ces extraits de scènes permet une discrimination claire entre la couverture végétale non brûlée en vert (figure 3g), les surfaces totalement brûlées en marron (figure $3 \mathrm{~h}$ ) et les surfaces brûlées cartographiées en rouge (figure $3 \mathrm{i}$ ). Cette interprétation permet de mettre en exergue, la potentialité des images S-2 pour la détection et la cartographie des surfaces brûlées. Il découle de ces extraits de scènes que les images $\mathrm{S}-2$ disposent du potentiel pour la restitution de la couverture végétale verte, des surfaces brûlées et aussi d'autres éléments de surface utiles pour la mise en œuvre des mesures de protection. Il s'agit notamment du relief, des habitations, des zones de culture, des routes et même de petites pistes identifiables sur les scènes présentées (figure $3 \mathrm{~g}$ et $3 \mathrm{~h}$ ).

L'étude a également permis de cartographier deux types de feux de végétation, les feux utilitaires et les feux incontrôlés. Les figures $4 a$ et $4 b$ représentent respectivement des extraits des feux utilitaires (inférieurs à 0,5 ha) et des feux incontrôlés (supérieurs ou égaux à 0,5 ha).

\section{Distribution spatiale des feux utilitaires}

La distribution spatiale des feux utilitaires et le couvert végétal brûlé sont représentés par la figure 5. L'analyse qualitative permet de déduire que les feux utilitaires s'opèrent sur toute l'étendue du territoire national et dans toutes les régions du pays (figure $5 \mathrm{a}$ ). Le couvert végétal brûlé par les feux utilitaires est composé essentiellement des cultures et jachères, savanes, forêts claires, forêts riveraines, parcs agroforestiers, zones marécageuses, forêts denses et plantations (figure $5 b$ ).

L'analyse quantitative montre que les surfaces brûlées par les feux utilitaires représentent $21,75 \%$, soit $1278,73 \mathrm{~km}^{2}$. La figure 6 illustre la répartition des superficies brûlées entre les différentes régions. Concernant les feux utilitaires, il découle des résultats que la région de la Kara, la région des Plateaux et la région Centrale enregistrent les valeurs les plus élevées. Les superficies brûlées correspondantes sont respectivement de $337,64 \mathrm{~km}^{2}, 316,13 \mathrm{~km}^{2}$ et de $301,71 \mathrm{~km}^{2}$ (respectivement $5,74 \%, 5,38 \%$ et 5,13\%). La région des Savanes et la région Maritime sont les moins touchées par les feux utilitaires (respectivement $1,95 \%$ et $3,55 \%$ ).

Les statistiques montrent également que le couvert le plus impacté par les feux utilitaires est composé essentiellement des cultures et jachères $(7,08 \%)$, savanes $(6,10 \%)$, forêts claires $(3,85 \%)$, forêts riveraines $(2,35 \%)$. Les parcs agroforestiers, les zones marécageuses, les forêts denses et les plantations sont moins touchés par les feux utilitaires avec des proportions de l'ordre de 0,34 \%, 0,47 \%, 0,48 \% et $1,09 \%$, respectivement (figure 7 ).

\section{Distribution spatiale des feux incontrôlés}
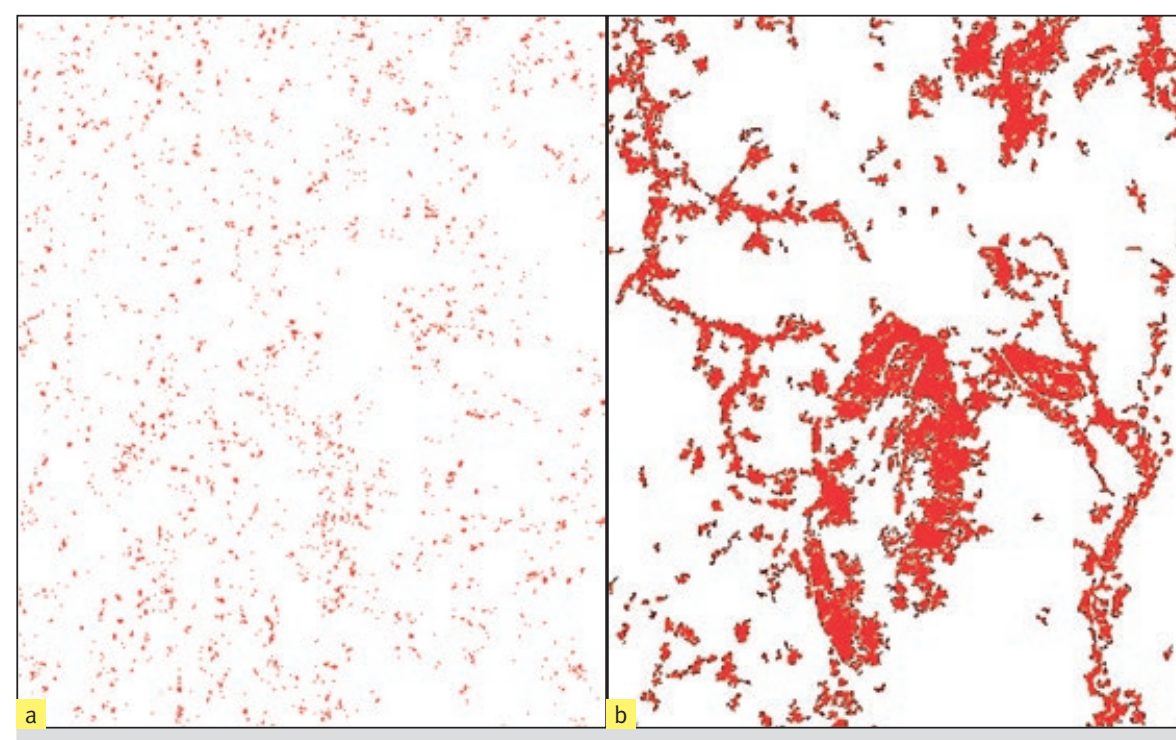

Figure 4.

Extraits des deux types de feux de végétation.

(a) Feux utilitaires (SB $<0,5$ ha).

(b) Feux incontrôlés ( $S B \geq 0,5$ ha).

Extracts from the two types of vegetation fires.

(a) Utility fires $(S B<0.5 \mathrm{ha})$.

(b) Uncontrolled fires $(S B \geq 0,5 \mathrm{ha})$.
La répartition géographique des feuxincontrôlés et le couvert végétal brûlé sont représentés dans la figure 8. L'interprétation visuelle a permis de constater que les feux incontrôlés s'opèrent sur toute l'étendue du territoire national avec une variété de densités dans les différentes régions du pays (figure $8 \mathrm{a}$ ). Le couvert végétal brûlé par les feux incontrôlés est composé essentiellement des savanes, cultures et jachères, plantations, forêts riveraines, forêts claires, forêts denses, parcs agroforestiers et zones marécageuses (figure 8b).

L'analyse statistique des surfaces brûlées par les feux incontrôlés montre qu'elles représentent $78,25 \%$, soit $4599,27 \mathrm{~km}^{2}$. La figure 6 retrace la répartition des feux incontrôlés par région. Les résultats révèlent que la région de la Kara et la région des Savanes sont les plus exposées à ces feux. Les deux régions enregistrent respectivement des superficies brûlées de $1491,56 \mathrm{~km}^{2}$ et de $1317,54 \mathrm{~km}^{2}$ correspondant respectivement à $25,38 \%$ et à $22,41 \%$. La région Centrale et la région des Plateaux sont 
Bois et Forêts des Tropiques - ISSN: L-0006-579X

Volume $347-1^{\text {st }}$ quarter - February 2021 - p. 59-75

70 FOCUS / BUSH FIRES DETECTION WITH SENTINEL-2

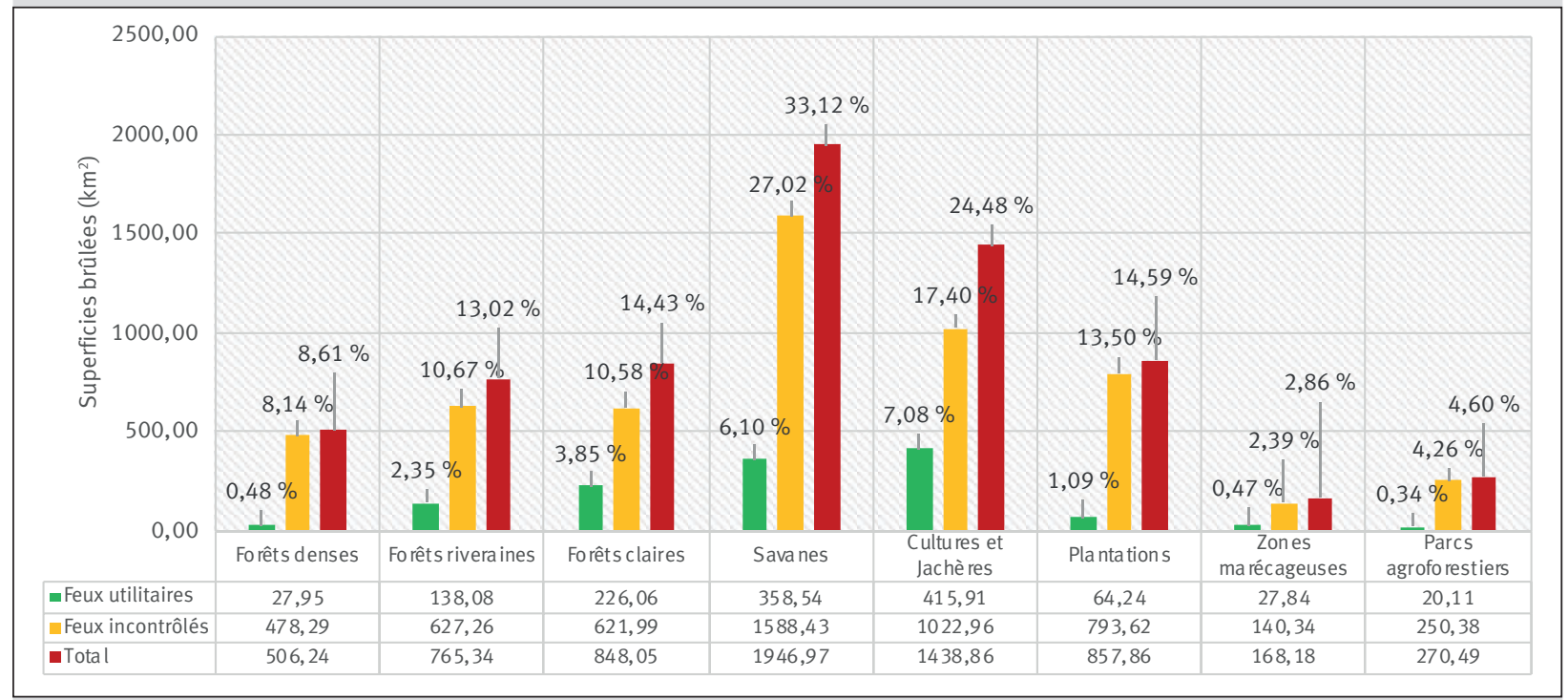

Figure 7.

Répartition de l'ensemble des superficies du couvert végétal brûlé par les feux utilitaires (FU) et les feux incontrôlés (FI). Distribution of the total area of vegetation cover burned by utility fires (FU) and uncontrolled fires (FI).

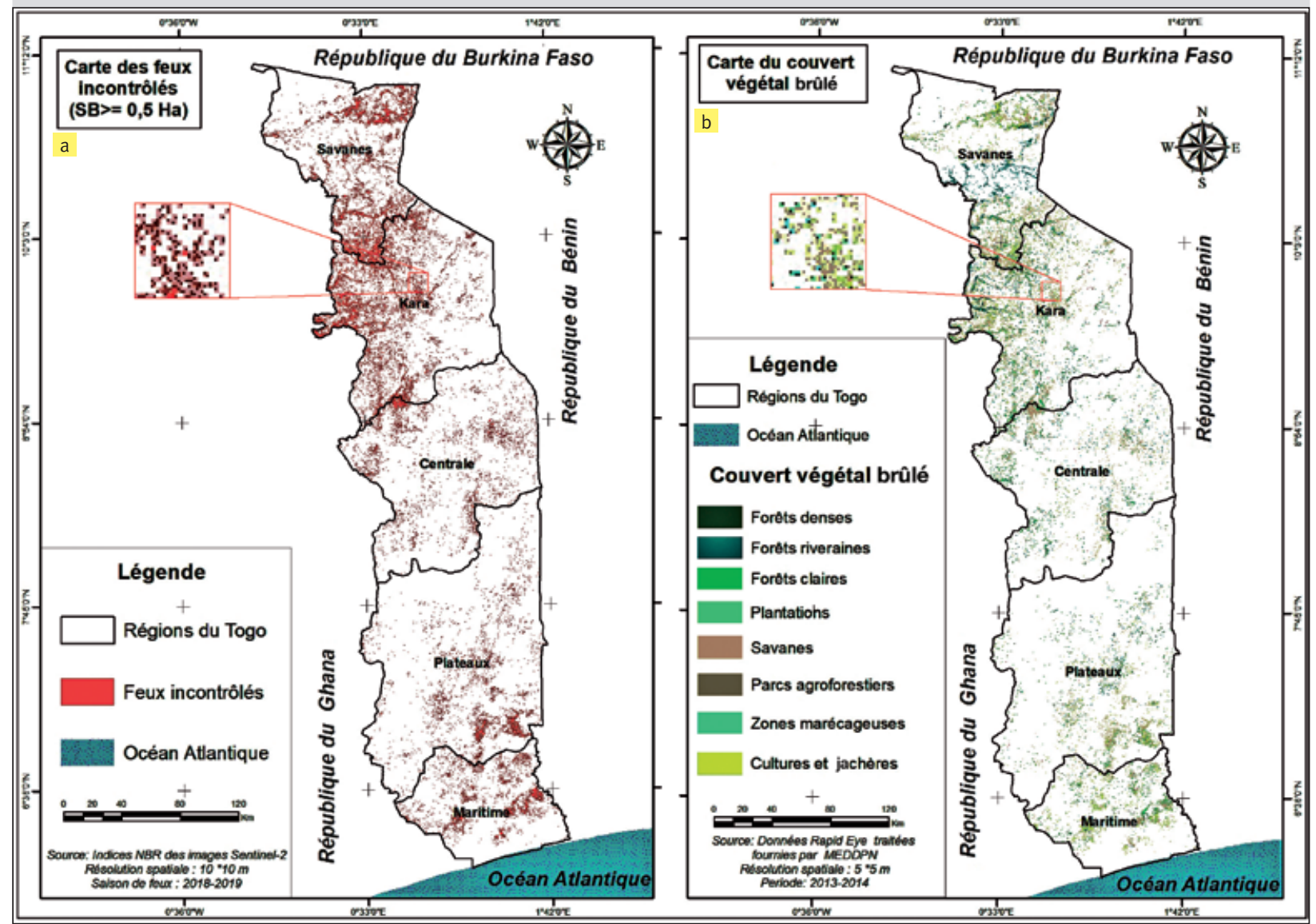

Figure 8.

Distribution spatiale des feux incontrôlés. (a) Carte des feux incontrôlés. (b) Carte du couvert végétal brûlé.

Spatial distribution of uncontrolled fires. (a) Map of uncontrolled fires (in red). (b) Map of burnt vegetation cover. 
Distribution spatiale de l'ensemble des surfaces brûlées

Les distributions spatiales de l'ensemble des surfaces brûlées (feux utilitaires et feux incontrôlés) et de l'ensemble du couvert végétal brûlé pour la saison des feux 2018-2019 sont représentées dans la figure 9. Il découle de l'analyse visuelle que les feux de végétation se produisent sur toute l'étendue du territoire national (figure 9a) et affectent une multitude de classes du couvert végétal (figure 9b).

Les statistiques des surfaces touchées par l'ensemble des feux de végétation sont consignées dans la figure 6 . À l'échelle nationale, les feux utilitaires représentent 21,75\% des surfaces alors que les incendies de forêts en concernent $78,25 \%$. Sur le plan régional, la région de la Kara et la région des Savanes restent les plus exposées à ce phénomène. Les deux régions enregistrent des superficies brûlées de $1829,20 \mathrm{~km}^{2}$ et de $1431,89 \mathrm{~km}^{2}$, respectivement. Les pourcentages correspondants sont de $31,12 \%$ et $24,36 \%$. La région Centrale et la région des Plateaux sont moyennement touchées avec des pourcentages de $16,92 \%$ et de $16,09 \%$ respectivement. La région Maritime est faiblement touchée par les feux $(11,51 \%)$. Les superficies du couvert végétal brûlées par l'ensemble des feux apparaissent dans la figure 7 . Les résultats montrent que les savanes, les cultures et jachères sont les plus impactées par l'ensemble des feux (33,12\% et 24,48\%, respectivement). Le couvert végétal moyennement touché est composé des plantations $(14,59 \%)$, des forêts claires $(14,43 \%)$ et des forêts riveraines $(13,02 \%)$. Le couvert végétal faiblement affecté concerne les forêts denses $(8,61 \%)$, les parcs agroforestiers $(4,60 \%)$ et les zones marécageuses (2,86\%).

\section{Discussion}

L'interprétation visuelle des images S-2 en coloration naturelle a permis de montrer le potentiel des images S-2 pour la détection des surfaces brûlées. Il découle des analyses que les images S-2 sont d'une qualité satisfaisante pour permettre une bonne visualisation de la végétation non brûlée et des surfaces brûlées. Selon les travaux de recherche de Konko et al. (2018b) et de Labib et Harris (2018), les capteurs MSI du satellite Sentinel-2 présentent de bonnes performances dans la restitution des scènes avec une amélioration de la lisibilité des images et de la résolution spatiale par rapport à d'autres systèmes imageurs fournissant des données similaires à accès libre, notamment le système Landsat.

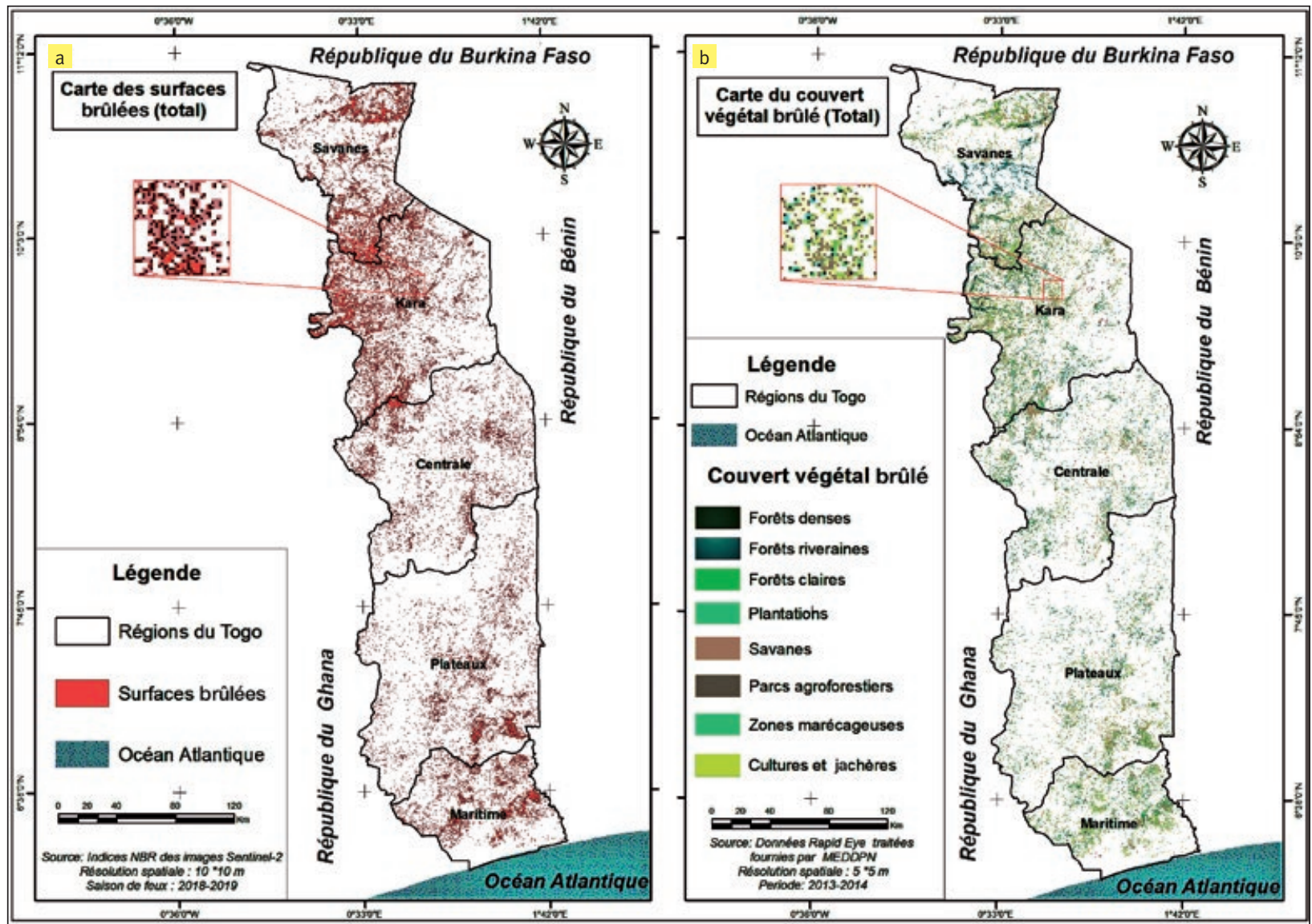

Figure 9.

Distribution spatiale de l'ensemble des feux de végétation. (a) Carte des surfaces brûlées. (b) Carte du couvert végétal brûlé. Spatial distribution of all vegetation fires. (a) Map of burnt surfaces. (b) Map of burnt vegetation cover. 
Les résultats sur la potentialité des images S-2 dans la restitution des ressources en eau et la création de masque d'eau sont encourageants. Ces performances ont été également discutées dans les travaux de recherche de Konko et al. (2018a) en contexte tropical, dont les auteurs ont conclu que les images S-2 disposent d'une grande potentialité pour la discrimination des eaux de surface à partir de l'indice NDWI. Toutefois, ces auteurs soulèvent le problème des nuages, qui constituent une contrainte majeure en contexte tropical.

L'indice $\triangle$ NBR obtenu dans le cadre de cette étude est d'une bonne qualité, permettant ainsi une cartographie de qualité des surfaces brûlées. Selon Keeley (2009), le $\Delta$ NBR est un excellent indicateur pour l'identification des zones brûlées et la mesure des brûlages. Les résultats obtenus avec l'indice $\triangle N B R$ témoignent de la qualité des données S-2. Ces données présentent, en effet, de bonnes performances avec une bonne lisibilité des scènes pour l'identification des surfaces brûlées.

Outre la qualité des images S-2, la méthode de l'indice NBR utilisée a joué également un rôle essentiel dans l'extraction précise des surfaces brûlées suivant les classes d'occupation du sol. La précision globale des résultats obtenus est de l'ordre de $92,53 \%$. À l'exception de la classe cultures et jachères, les précisions F-Score sont supérieures à $90 \%$ pour l'ensemble des classes d'occupation du sol. Il découle de ces indicateurs de précision que la méthode d'analyse est bonne et que les résultats sont exploitables. Les précisions obtenues pour cette étude sont similaires à celles fournies par Mpakairi et al. (2020) au Zimbabwe. En effet, ces auteurs ont aussi utilisé l'indice NBR et ont trouvé une précision de l'ordre de $91 \%$ pour les classes de forêts et de $76 \%$ pour la classe des arbustes. Cette classe présente les mêmes caractéristiques que la classe des cultures et jachères dont la précision F-Score est de 87,39 \% dans le cadre de la présente étude. De plus, la performance de l'indice NBR varie suivant les types d'occupation du sol. L'indice NBR est moins performant pour les classes les moins densément couvertes de végétation. Il y a donc nécessité d'utiliser d'autres indices spectraux prenant en compte la réflectance du sol pour la cartographie des surfaces brûlées des classes les moins couvertes par la végétation.

Les statistiques à l'échelle nationale des surfaces brûlées par l'ensemble des feux de végétation montrent que les feux utilitaires représentent seulement $21,75 \%$ des superficies contre 78,25 \% pour les feux incontrôlés. Au Togo, les feux utilitaires sont courants pour le nettoiement des champs, le pastoralisme et le renouvellement de la paille. Le faible pourcentage des surfaces brûlées par ces feux utilitaires s'explique par le fait qu'ils échappent souvent au contrôle humain, gagnent du terrain et deviennent des feux incontrôlés. Le taux élevé des surfaces brûlées par les feux incontrôlés confirme la difficulté de maîtrise des feux utilitaires. Sur le plan régional, la région de la Kara et la région des Savanes restent les plus exposées aux feux. Ceci pourrait s'expliquer par la sévérité de la saison sèche dans les régions septentrionales et aussi par la structure et la composition du paysage.

En ce qui concerne le couvert végétal brûlé pour l'ensemble des feux, les résultats révèlent que les savanes, les cultures et jachères sont les plus impactées (33,12\%

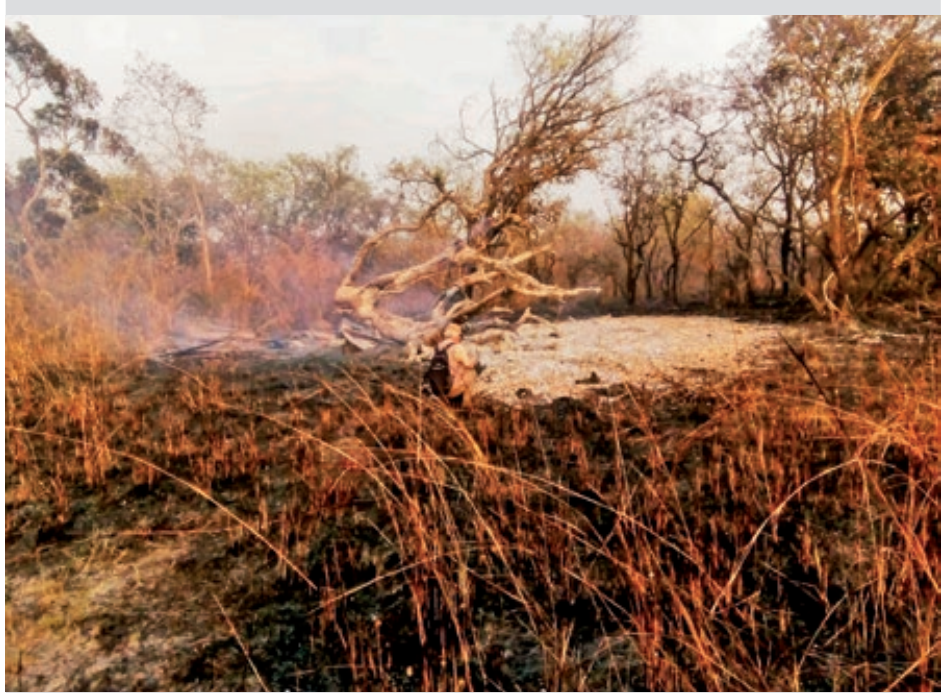

Photo 2.

Aperçu d'une zone savanicole brûlée pour le renouvellement de la paille dans la région Centrale.

Overview of a burnt savannah area for straw renewal in the Central region.

Photo Y. Konko.

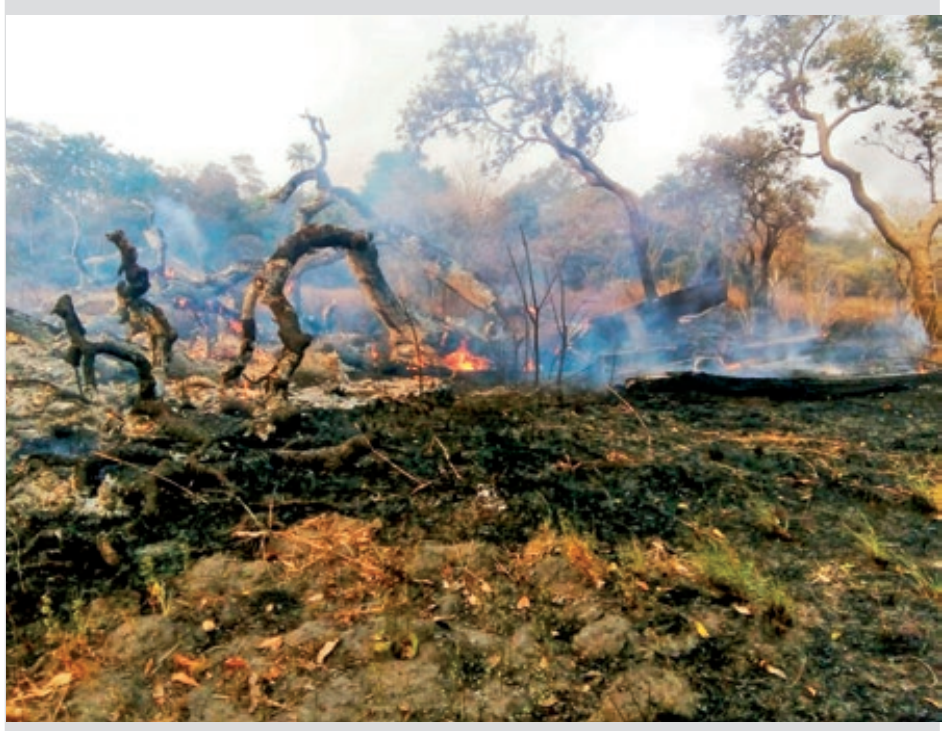

Photo 3.

Vue d'une portion de la forêt communautaire d'Alibi brûlée dans la région Centrale.

View of a portion of the burnt Alibi community forest in the Central region.

Photo Y. Konko.

et $24,48 \%$, respectivement). Les pourcentages élevés de ces formations brûlées s'expliquent par le fait qu'elles contiennent beaucoup d'herbacées, couvrent de vastes étendues et sont omniprésentes dans toutes les régions du pays. En saison sèche, un petit feu permet d'embraser une grande surface. En termes d'utilisation, les habitants mettent le feu dans les savanes pour l'agriculture, pour la chasse des animaux sauvages et pour le renouvellement de la paille pour le pastoralisme. La photo 2 est une vue d'une zone savanicole brûlée pour le renouvellement de la paille dans la région Centrale. Dans les zones de cultures et jachères, les populations locales ont recours au feu pour le défrichement et le nettoiement des champs. 
Les résultats de cette étude montrent également que les forêts denses (8,61\%), les parcs agroforestiers (4,60\%) et les zones marécageuses $(2,86 \%)$ sont faiblement affectés par les feux. S'agissant des forêts denses, elles sont peu représentées à l'échelle nationale, et sont souvent l'objet de lieux de culte et ainsi protégées par la population (Kokou et Sokpon, 2006 ; Konko et al., 2018b). La strate inférieure des forêts denses est également dépourvue d'herbacées, rendant difficile la propagation des feux. Les feux observés dans des forêts denses sont, dans la plupart des cas, des feux sauvages. La photo 3 est un aperçu d'une portion de la forêt communautaire d'Alibi brûlée dans la région Centrale.

Le pourcentage faible des parcs agroforestiers et des zones marécageuses brûlés ne signifie pas que ces formations ne sont pas touchées par les feux, mais plutôt qu'elles sont localisées dans certaines régions et que les statistiques présentées ici le sont à l'échelle nationale. En effet, les parcs agroforestiers brûlés se rencontrent beaucoup plus dans la région des Savanes alors que les zones marécageuses brûlées en saison sèche sont prépondérantes dans la région Maritime. Les deux types de formations sont exposés aux feux de végétation.

L'ensemble des feux de végétation enregistrés au Togo pour la saison des feux 2018-2019 a permis le brûlage de $5877,99 \mathrm{~km}^{2}$ du couvert végétal, soit 10,39 \% du territoire national. Ces feux de brousse se répercutent négativement sur les efforts de conservation et de restauration de la biodiversité augmentant ainsi les émissions de gaz à effet de serre. Selon certains auteurs, ils dégradent les écosystèmes, modifient le paysage et perturbent la biocénose et l'écologie des populations (Bowman et al., 2009 ; Yue et al., 2015 ; Lewis et al., 2015 ; Konko, 2016).

\section{Conclusion}

La présente étude a permis d'explorer la potentialité des images S-2 pour la détection des surfaces brûlées, la cartographie des feux utilitaires et des feux incontrôlés en Afrique tropicale. Elle a également testé la performance de la méthode de cartographie des feux de végétation à partir de l'indice NBR initialement conçu pour les images Landsat et évalué la biomasse végétale brûlée. Les résultats révèlent que les images Sentinel-2 présentent du potentiel dans la restitution des surfaces brûlées. La performance de la méthode de l'indice NBR sur les images S-2 est satisfaisante. La cartographie des feux de végétation montre que les surfaces brûlées par les feux utilitaires représentent $21,75 \%$ contre $78,25 \%$ pour les feux incontrôlés. L'ensemble des feux de végétation enregistrés au Togo pour la saison des feux 2018-2019 a malheureusement occasionné la perte de $5878 \mathrm{~km}^{2}$ du couvert végétal composé majoritairement des savanes (33,12\%), des cultures et jachères $(24,48 \%)$, des plantations $(14,59 \%)$, des forêts claires $(14,43 \%)$ et des forêts riveraines $(13,02 \%)$. Ces feux de végétation se répercutent négativement sur les efforts de conservation et de restauration de la biodiversité augmentant ainsi les émissions atmosphériques des gaz à effet de serre, et contribuant finalement au réchauffement climatique. Les résultats obtenus constituent des éléments tangibles pour le suivi, la sensibilisation, l'élaboration des plans d'aménagement, de prévention et de gestion des feux. En bref, pour la mise en œuvre des mesures d'adaptation et d'atténuation.

\section{Remerciements}

Cette recherche n'a reçu aucune subvention spécifique de la part des organismes du secteur public et commercial. Nous remercions l'ONG APEDD Togo qui nous a accordé son soutien pour les investigations sur le terrain. Merci également à M. NANGUE Kombaté Bouaman Gaston pour sa contribution dans la collecte des données de terrain.

\section{Références}

Afelu B., 2016. Contribution à la gestion des feux de végétation au Togo. Thèse de doctorat, Université de Lomé, Togo, 177 p.

Atsri H. K., Konko Y., Cuni-Sanchez A., Abotsi K. E., Kokou K., 2018. Changes in the West African forest-savanna mosaic, insights from central Togo. Plos One, 13v (10): e0203999. https://doi.org/10.1371/journal.pone.0203999

Bartalev S. A., Egorov V. A., Loupian E. A., Uvarov I. A., 2007. Multi-year circumpolar assessment of the area burnt in boreal ecosystems using SPOT-VEGETATION. International Journal of Remote Sensing, 28 (6): 1397-1404. https://doi. org/10.1080/01431160600840978

Bastarrika A., Chuvieco E., Pilar Martín M., 2011. Mapping burned areas from Landsat TM/ETM+ data with a two-phase algorithm: Balancing omission and commission errors. Remote Sensing of Environment, 115 (4): 1003-1012. https://doi. org/10.1016/i.rse.2010.12.005

Boschetti M., Stroppiana D., Brivio P. A., 2010. Mapping burned areas in a Mediterranean environment using soft integration of spectral indices from high resolution satellite images. Earth Interactions, 14 (17): 1-20. https://doi.org/10.1175/2010El349.1 Bowman D. M. J. S., Balch J. K., Artaxo P., Bond W. J., Carlson J. M., Cochrane M. A., et al., 2009. Fire in the Earth system. Science, 324 (5926): 481-484. https://doi.org/10.1126/ science.1163886

Chuvieco E., Martin M. P., Palacios A., 2002. Assessment of different spectral indices in the red-near-infrared spectral domain for burned land discrimination. International Journal of Remote Sensing, 23 (23): 5103-5110. https://doi. org/10.1080/01431160210153129

Chuvieco E., Englefield P., Trischenko A., Lio Y., 2008. Generation of long time series of burn area maps of the boreal forest from NOAA-AVHRR composite data. Remote Sensing of Environment, 112 (5): 2381-2396. https://doi.org/10.1016/j. rse.2007.11.007

Chuvieco E., Aguado I., Yebra M., Nieto H., Salas J., Pilar Martín M., et al., 2010. Development of a framework for fire risk assessment using remote sensing and geographic information system technologies. Ecological Modelling, 221 (1): 46-58. https://doi. org/10.1016/i.ecolmodel.2008.11.017

Chuvieco E., Lizundia-Loiola J., Pettinari M. L., Ramo R., Padilla M., Tansey K., Mouillot F., et al., 2018. Generation and analysis of a new global burned area product based on MODIS $250 \mathrm{~m}$ reflectance bands and thermal anomalies. Earth System Science Data, 10 (4): 2015-2031. https://doi.org/10.5194/ essd-10-2015-2018

Chuvieco E., Mouillot F., Van der Werf G. R., San Miguel J., Tanase M., Koutsias N., et al., 2019. Historical background and current developments for mapping burned area from satellite Earth observation. Remote Sensing of Environment, 225 (1): 45-64. https://doi.org/10.1016/i.rse.2019.02.013 
Congalton R. G., 1991. A review of assessing the accuracy of classifications of remotely sensed data. Remote Sensing of Environment, 37 (1): 35-46. https://doi. org/10.1016/0034-4257(91)90048-B

Doerr S. H., Shakesby R. A., Blake W. H., Chafer C. J., Humphreys G. S., Wallbrink P. J., 2006. Effects of differing wildfire severities on soil wettability and implications for hydrological response. Journal of Hydrology, 319 (1-4): 295-311. https://doi. org/10.1016/i.jhydrol.2005.06.038

Ern H., 1979. Die Vegetation Togos. Gliederung, Gefährdung, Erhaltung. Willdenowia, 9: 295-312. https://www.jstor.org/ stable/3995654?seq=1

García-Llamas P., Suárez-Seoane S., Fernández-Guisuraga J. M., Fernández-García V., Fernández-Manso A., Quintano C., et al. 2019. Evaluation and comparison of Landsat 8, Sentinel-2 and Deimos-1 remote sensing indices for assessing burn severity in Mediterranean fire-prone ecosystems. International Journal of Applied Earth Observation and Geoinformation, 80: 137-144. https://doi.org/10.1016/i.jag.2019.04.006

González F. E., Ruiz J. M., Acosta F. M., 2013. Manuel de télédétection spatiale. TELECAN. Université de Las Palmas, Gran Canaria, 337 p. https://www.grss-ieee.org/wp-content/ uploads/2014/07/FR TUTORIAL COMPLETO.pdf

Guelly K. A., 1994. Les savanes de la zone forestière subhumide du Togo. Thèse de doctorat. Université Pierre Marie-Curie, Paris VI, France. http://www.theses.fr/1994PA066589

IPCC, 2018. Global warming of $1.5^{\circ} \mathrm{C}$ : An IPCC Special Report on the impacts of global warming of $1.5^{\circ} \mathrm{C}$ above pre-industrial levels and related global greenhouse gas emission pathways, in the context of strengthening the global response to the threat of climate change, sustainable development, and efforts to eradicate poverty. Cambridge, UK, Cambridge University Press, 562 p. https://www.ipcc.ch/sr15/

Kamau P. N., Medley K. E., 2014. Anthropogenic fires and local livelihoods at Chyulu Hills, Kenya. Landscape and Urban Planning, 124: 76-84. https://doi.org/10.1016/i. landurbplan.2014.01.010

Keeley J. E., 2009. Fire intensity, fire severity and burn severity: a brief review and suggested usage. International Journal of Wildland Fire, 18 (1): 116-126. https://doi.org/10.1071/ WF07049

Key C., Benson N., 2006. Landscape assessment: Ground measure of severity, the Composite Burn Index, and remote sensing of severity, the Normalized Burn Index. In: Lutes D., Keane R., Caratti J., Key C., Benson N., Sutherland S., et al. (eds). FIREMON: Fire effects monitoring and inventory system. Fort Collins, CO, USA, USDA Forest Service, Rocky Mountains Research Station, General Technical Report RMRS-GTR-164-CD, LA 1-51. https://www.fs.fed.us/rm/pubs/rmrs gtr164.pdf

KnorrW., Jiang L., Arneth A., 2016. Climate, $\mathrm{CO}_{2}$ and human population impacts on global wildfire emissions. Biogeosciences, 13 (1): 267-282. https://doi.org/10.5194/bg-13-267-2016

Kokou K., Sokpon N., 2006. Les forêts sacrées du couloir du Dahomey. Bois et Forêts des Tropiques, 288 : 15-23. https:// revues.cirad.fr/index.php/BFT/article/view/20312

Konko Y., 2016. Contribution of Remote Sensing and GIS to the Integrated Management of Community Forest Resources in the Bas-Mono Valley (South-East Togo). Master's Thesis, Post University Regional School of Integrated Management of Tropical Forests and Territories, Kinshasa, Democratic Republic of Congo, 94 p. https://doi.org/10.13140/RG.2.2.20280.70403
Konko Y., Bagaram B., Julien F., Akpamou K. G., Kokou K., 2018a. Multitemporal Analysis of Coastal Erosion Based on Multisource Satellite Images in the South of the Mono Transboundary Biosphere Reserve in Togo (West Africa). Open Access Library Journal, 5 (4): e4526. https://doi.org/10.4236/oalib.1104526

Konko Y., Rudant J. P., Akpamou G. K., Noumonvi K. D., Kokou K., 2018b. Spatio-Temporal Distribution of Southeastern Community Forests in Togo (West Africa). Journal of Geoscience and Environment Protection, 6 (7): 51-65. https://doi.org/10.4236/ gep.2018.67004

Konko Y., Okhimambe A., Nimon P., Asaana J., Rudant J. P., Kokou K., 2020. Coastline Change Modelling Induced by Climate Change Using Geospatial Techniques in Togo (West Africa). Advances in Remote Sensing, 9 (2): 85-100. https://doi. org/10.4236/ars.2020.92005

Labib S. M., Harris A., 2018. The potentials of Sentinel-2 and LandSat- 8 data in green infrastructure extraction, using object based image analysis (OBIA) method. European Journal of Remote Sensing, 51 (1): 231-240. https://doi.org/10.1080/22 797254.2017.1419441

Lasaponara R., 2006. Estimating spectral separability of satellite derived parameters for burned areas mapping in the Calabria region by using SPOT-vegetation data. Ecological Modelling, 196 (1-2): 265-270. https://doi.org/10.1016/j. ecolmodel.2006.02.025

Lewis S. L., Edwards D. P., Galbraith D., 2015. Increasing human dominance of tropical forests. Science, 349 (6250): 827-832. https://doi.org/10.1126/science.aaa9932

Mallinis G., Mitsopoulos I., Chrysafi I., 2018. Evaluating and comparing Sentinel $2 \mathrm{~A}$ and Landsat- 8 Operational Land Imager (OLI) spectral indices for estimating fire severity in a Mediterranean pine ecosystem of Greece. GIScience and Remote Sensing, 55 (1): 1-18. https://doi.org/10.1080/15481603.2017.1 354803

McFeeters S. K., 1996. The Use of the Normalized Difference Water Index (NDWI) in the Delineation of Open Water Features. International Journal of Remote Sensing, 17 (7): 1425-1432. https://doi.org/10.1080/01431169608948714

Miller J. D., Knapp E. E., Key C. H., Skinner C. N., Isbell C. J., Creasy R. M., et al., 2009. Calibration and validation of the relative differenced Normalized Burn Ratio (RdNBR) to three measures of fire severity in the Sierra Nevada and Klamath Mountains, California, USA. Remote Sensing of Environment, 113 (3) : 645-656. https://doi.org/10.1016/j.rse.2008.11.009

Mpakairi K. S., Ndaimani H., Kavhu B., 2020. Exploring the utility of Sentinel-2 MSI derived spectral indices in mapping burned areas in different land-cover types. Scientific African, 10: e00565. https://doi.org/10.1016/j.sciaf.2020.e00565

Nimon P., Issaou L., Konko Y., Kokou K., 2020. Spatio-Temporal Patterns of Rainfall Variability for Wet Season over Togo in West Africa. Open Access Library Journal, 7 (1): e6044. https://doi. org/10.4236/oalib.1106044

Reid C. E., Brauer M., Johnston F. H., Jerrett M., Balmes J. R., Elliott C. T., 2016. Critical review of health impacts of wildfire smoke exposure. Environmental Health Perspectives, 124 (9): 1334-1343. https://doi.org/10.1289/ehp.1409277

Rwanga S. S., Ndambuki J. M., 2017. Accuracy assessment of land use/land cover classification using remote sensing and GIS. International Journal of Geosciences, 8 (4): 611-622. https://doi.org/10.4236/iig.2017.84033 
Smith A. M. S., Drake N. A., Wooster M. J., Hudak A. T., Holden Z. A., Gibbons C. J., 2007. Production of Landsat ETM+ reference imagery of burned areas within Southern African savannahs: Comparison of methods and application to MODIS. International Journal of Remote Sensing, 28 (12): 2753-2775. https://doi. org/10.1080/01431160600954704

Schroeder Th., Behnert I., Schaale M., Fischer J., Doerffer R., 2007. Atmospheric correction algorithm for MERIS above case-2 waters. International Journal of Remote Sensing, 28 (7): 14691486. https://doi.org/10.1080/01431160600962574

Schroeder W., Oliva P., Giglio L., Quayle B., Lorenz E., Morelli F., 2016. Active fire detection using Landsat-8/OLI data. Remote Sensing of Environment, 185: 210-220. https://doi. org/10.1016/j.rse.2015.08.032

Sow M., Hély C., Mbow C., Sambou B., 2013. Fuel and fire behaviour analysis for early-season prescribed fire planning in Sudanian and Sahelian savannas. Journal of Arid Environments, 89: 84-93. https://doi.org/10.1016/i.jaridenv.2012.09.007

Smith A. M. S., Drake N. A., Wooster M. J., Hudak A. T., Holden Z. A., Gibbons C. J., 2007. Production of Landsat ETM+ reference imagery of burned areas within Southern African savannahs: Comparison of methods and application to MODIS. International Journal of Remote Sensing, 28 (12): 2753-2775. https://doi. org/10.1080/01431160600954704

Stehman S. V., Foody G. M., 2019. Key issues in rigorous accuracy assessment of land cover products. Remote Sensing of Environment, 231: 111199. https://doi.org/10.1016/j. rse.2019.05.018

Story M., Congalton R. G., 1986. Accuracy assessment: a user's perspective. Photogrammetric Engineering and Remote Sensing, 52 (3): 397-399. https://www.asprs.org/wp-content/ uploads/pers/1986journal/mar/1986 mar 397-399.pdf

Stroppiana D., Boschetti M., Zaffaroni P., Brivio P., 2009. Analysis and interpretation of spectral indices for soft multicriteria burned-area mapping in Mediterranean regions. IEEE Geoscience and Remote Sensing Letters, 6 (3): 499-503. https://doi. org/10.1109/LGRS.2009.2020067

Tucker C. J., 1979. Red and photographic infrared linear combinations for monitoring vegetation. Remote Sensing of Environment, 8: 127-150. https://doi.org/10.1016/0034-4257(79)90013-0 Veraverbeke S., Verstraeten W. W., Lhermitte S., Goossens R., 2010a. Evaluating Landsat Thematic Mapper spectral indices for estimating burn severity of the 2007 Peloponnese wildfires in Greece. International Journal of Wildland Fire, 19 (5): 558569. https://doi.org/10.1071/WF09069.

Veraverbeke S., Verstraeten W. W., Lhermitte S., Goossens R., 2010b. Illumination effects on the differenced Normalized Burn Ratio's optimality for assessing fire severity. International Journal of Applied Earth Observation and Geoinformation, 12 (1): 60-70. https://doi.org/10.1016/i.jag.2009.10.004

Veraverbeke S., Hook S., Hulley G., 2012. An alternative spectral index for rapid fire severity assessments. Remote Sensing of Environment, 123: 72-80. https://doi.org/10.1016/i. rse.2012.02.025

Verhegghen A., Eva H., Ceccherini G., Achard F., Gond V., Gourlet-Fleury S., et al., 2016. The potential of Sentinel satellites for burnt area mapping and monitoring in the Congo Basin forests. Remote Sensing, 8 (12): 986. https://doi.org/10.3390/ rs8120986
Wang X., Liu Y., Ling F., Liu Y., Fang F., 2017. Spatio-Temporal Change Detection of Ningbo Shoreline Using Landsat Time-Series Images during 1976-2015. ISPRS International Journal of Geo-Information, 6 (3): 68. https://doi.org/10.3390/ iigi6030068

Yue C., Ciais P., Cadule P., Thonicke K., van Leeuwen T. T., 2015. Modelling the role of fires in the terrestrial carbon balance by incorporating SPITFIRE into the global vegetation model ORCHIDEE. Part 2: Carbon emissions and the role of fires in the global carbon balance. Geoscientific Model Development, 8 (5): 1285-1297. https://doi.org/10.5194/gmd-8-1321-2015

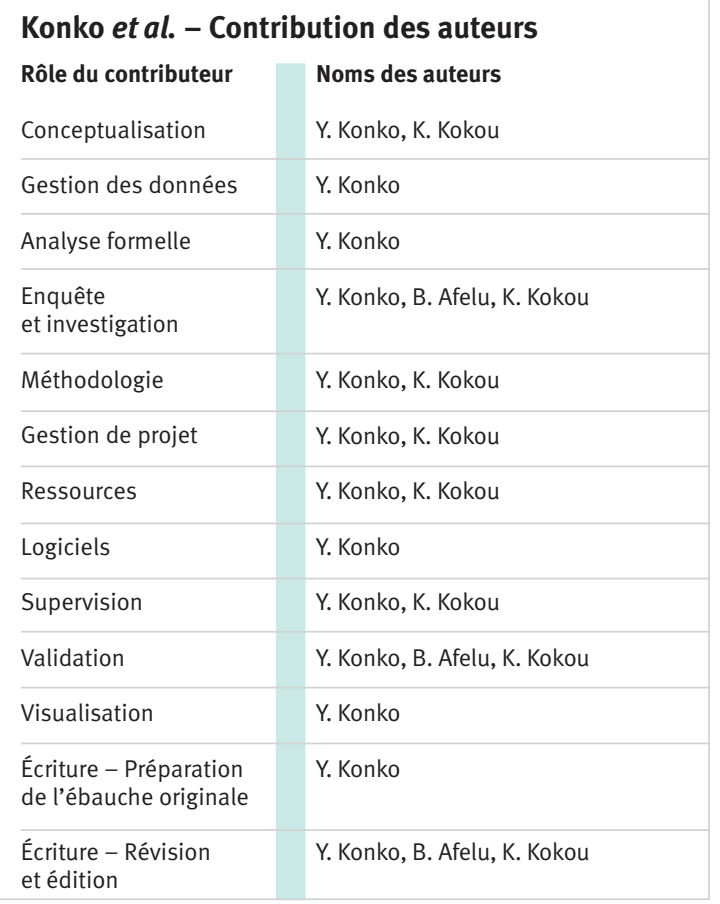
(c) Bois et Forêts des Tropiques (c) Cirad
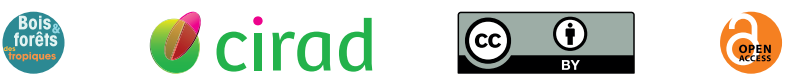

Cirad - Campus international de Baillarguet, 34398 Montpellier Cedex 5, France - Contact : bft@cirad.fr - ISSN : L-0006-579X 